\title{
Qui sont les personnes trans* et pourquoi ne les voit-on pas davantage participer au marché du travail
}

\author{
Dr François Bernard Malo
}

RÉSUMÉ. Cet article présente une définition large et inclusive de ce que sont les personnes trans*. Ancrée autour de quatre repères conceptuels majeurs (le sexe biologique, l'identité de genre, l'expression de genre et l'orientation sexuelle), cette définition est par la suite contextuali sée à l'aide de quatre indicateurs sociaux que sont l'âge, l'origine nationale/couleur de la peau/religion, la strate sociale et l'apparence générale. La prise en compte de l'ensemble de ces éléments permet au lecteur de mieux comprendre les raisons pour lesquelles les personnes trans* sont l'objet de tant d'incompréhensions et de violences. L'article se conclut enfin par la présentation d'un ensemble de propositions concrètes à l'intention des États, des Employeurs et des Syndicats afin que les droits des personnes trans* soient davantage reconnus sur le marché du travail et de l'emploi.

\begin{abstract}
$\boldsymbol{A B S T R A C T}$. This article begins by the presentation of a large and inclusive definition of what are the trans* people. Built around the notions of biological sex, gender identity, gender expression and sexual orientation, this definition is then placed in relation to other important social markers (age, national origin/skin colour/religion, social strata and gen eral appearance). All those variables are mobilized to help the reader understand the reasons why trans* people so frequently face misunderstanding and violence. This article concludes with pragmatic propositions suggested to States, Employers and Unions aimed at encouraging them to do all they can to make sure the rights of the trans* people are better respected on the labor and employment market.
\end{abstract}

\section{Introduction}

Le Québec est de plus en plus reconnu sur la scène internationale comme une société inclusive et l'un des plus importants leaders en matière de défense et de promotion des droits des personnes lesbiennes, gaies, bisexuelles, trans* et queer (LGBTQ). Après avoir créé en 2011 un Bureau de lutte contre l'homophobie, le gouvernement du Québec a accordé, en juin 2018, une subvention de quatre millions de dollars sur cinq ans afin de mettre en place un réseau international francophone en faveur de la protection des droits des personnes issues de la diversité sexuelle et de genre (Ouellette-Vézina, 2018). Pourtant, la réalité des personnes trans* n'est toujours pas très bien connue et de nombreux mythes et fausses conceptions

${ }^{\text {a }}$ Professeur (GRH-UT1), Université Laval

Revue Organisations \& Territoires $•$ Volume $27 \cdot \mathrm{N}^{\circ}{ }_{2} \cdot 2018$ continuent de se propager à leur égard (EricksonSchroth et Jacobs, 2017).

En se concentrant de manière spécifique sur la question des personnes trans*, cet article souligne qu'il n'existe pas, à l'heure actuelle, de définition communément admise de ce qu'est une personne trans*. En fait, il est même possible d'affirmer que la définition variera grandement selon la personne à qui l'on s'adresse et, entre autres choses, au lieu et à l'époque où la question est elle-même posée (Hines et Sanger, 2010). Comme nous aurons l'occasion de le remarquer, cette constatation porte en elle-même des implications colossales. En effet, selon la définition que l'on acceptera de considérer comme "vraie pour soi », les solutions que l'on privilégiera pour traiter de la problématique annoncée pourront prendre des directions totalement opposées ${ }^{1}$. 


\section{Notre définition des personnes trans*}

Rien dans la vie n'est à craindre, tout doit être compris. C'est maintenant le moment de comprendre davantage, afin de craindre moins.

- Marie Curie

Cherchant à prendre contre-pied à l'opinion d'un certain nombre de figures d'autorité qui ont généralement défini de manière infiniment simpliste ce que sont les personnes trans* (Salamon, 2010), nous dirons, aux fins de notre propos, qu'une personne trans* est une personne parfaitement saine de corps et d'esprit, dont l'identité de genre et/ou l'expression de genre ne correspondent pas, aux yeux de la société à l'intérieur de laquelle elle évolue à une époque donnée, au sexe qui lui a été assigné à la naissance (le plus souvent, par le médecin accoucheur ou la sage-femme) et aux normes de genre présentes dans cette société. Contrairement à ce que certaines conceptions simplistes pourraient nous faire croire, la personne trans* ne décide pas d'être ce qu'elle est. Pour paraphraser une célèbre chanson de Lady Gaga (Born This Way), elle est tout simplement "née ainsi », au fil du temps, et ne fait que « finir » par découvrir son véritable moi.

Volontairement large et inclusif, ce mot-valise regroupe, sans s'y limiter, les personnes se disant intersexuelles, transsexuelles, transgenres et travesties. Bien que ces trois derniers cas soient les éléments les plus visibles de la catégorie personnes "trans*», ce vocable inclut aussi, notamment, les personnes qui s’identifient "au-delà » des catégories usuelles (Harrington, 2016). À ce sujet, il est très important, de notre point de vue, que les personnes aient elles-mêmes l'occasion (si elles le souhaitent) de nous dire ce qu'elles sont et ne sont pas, à leurs propres yeux, et pourquoi elles se considèrent elles-mêmes comme des personnes trans*. Dit autrement, nous croyons que ce n'est pas à une forme quelconque d'autorité de dire "qui est trans* » et « qui ne l'est pas ».

Pour cette raison, évidemment, il est extrêmement difficile d'évaluer avec précision combien il y a de personnes trans* au sein de la population, car le processus de découverte et d'affirmation de soi des personnes trans* est parsemé d'embûches sociales, comme nous pourrons le constater. Alors que certains chiffres très conservateurs parlent d'un cas pour environ 30000 individus (soit des individus tellement perturbés par les pressions sociales liées à leur identité et/ou à leur expression de genre que leurs idées suicidaires les ont poussés à obtenir un « diagnostic formel » émis par une autorité médicale), d'autres avancent qu'il y aurait environ un cas pour 1500 individus (Conway, 2002).

En contexte d'interactions sociales habituelles, il nous semble important de préciser que nous pensons que c'est généralement manquer de délicatesse que de demander à une personne trans* si elle se définit comme une personne intersexuelle, transsexuelle, transgenre ou travestie. En effet, sur le marché du travail, nous considérons que cela est une question qui relève de l'intimité de chacun des individus et qu'elle n'a donc pas sa place. C'est, à notre avis, faire preuve d'une curiosité malsaine empreinte de voyeurisme que de chercher à en savoir plus.

Enfin, de nombreuses recherches empiriques ayant démontré qu'il n'y a aucune relation théorique ni pratique simple entre le sexe biologique, l'identité de genre, l'expression de genre et l'orientation sexuelle (du moins dans la plupart des sociétés examinées par les chercheurs) (Jordan-Young, 2016), l'expression "personnes trans* » exclut sciemment toute référence à l'orientation sexuelle. Ainsi, il peut donc y avoir, autant en pratique qu'en théorie, des personnes trans* hétérosexuelles, homosexuelles, bisexuelles, pansexuelles ou, encore, asexuelles. Comme nous le disions précédemment au sujet des distinctions « à ne pas imposer » lors de nos interactions sur le marché du travail avec une personne trans*, nous considérons qu'il est également inapproprié de lui demander quelle est son orientation sexuelle ou, pire encore, d'assumer automatiquement qu'elle est homosexuelle².

Pour bien comprendre cette définition, il faut savoir qu'elle réfère indirectement à quatre éléments catégoriels interreliés qui méritent d'être explorés plus à fond et qui doivent être compris comme faisant partie d'un continuum marqué, à chacune de ses extrémités, par les stéréotypes usuels faisant référence à ce que "devrait » être un homme ou une femme dans les sociétés occidentales contemporaines se disant les plus avancées d'un point de vue économique ${ }^{3}$ : le sexe biologique, l'identité de genre, l'expression de genre et enfin, l'orientation sexuelle ${ }^{4}$. 
Le sexe est généralement abordé par le biais de la biologie et il réfère à la fois aux organes sexuels externes et aux gènes qui y sont sous-jacents. Alors qu'à une extrémité on y retrouve « l'Homme », dont les gènes sont typiquement $\mathrm{XX}$, à l'autre extrémité nous y retrouvons la «Femme », dont les gènes sont typiquement XY. Bien que l'on en parle que très peu, il y a toutes sortes de variantes entre ces deux extrêmes (Ainsworth, 2015). Sans entrer dans ces multiples nuances, nous pouvons quand même prendre le temps de citer le cas des personnes dites intersexuelles ou encore hermaphrodites puis, ce qui est de plus en plus discuté au sein de la société québécoise, le cas des personnes transsexuelles qui se font opérer pour modifier leurs organes sexuels externes de telle sorte qu'ils ne correspondent plus à leurs gènes sous-jacents 5 .

L'identité de genre, quant à elle, est généralement envisagée du point de vue de la psychologie. Elle réfère à la façon dont la personne s'identifie ellemême sur le continuum du sexe biologique ${ }^{6}$. Alors que la plupart des personnes nées dans un corps d'homme s'identifieront à la catégorie "homme» et que la plupart des personnes nées dans un corps de femme auront tendance à s'identifier à la catégorie "femme", de nombreuses variantes sont encore une fois possibles. La plus connue de ces variantes est celle des personnes transgenres (Sironi, 2011). Il s'agit, ici, d'une personne qui s'identifie à un genre qui ne correspond pas, a priori, à son sexe biologique, mais qui ne ressent pas pour autant le besoin, notamment, de transformer ses organes sexuels externes au moyen de la chirurgie. Beaucoup de personnes transgenres, toutefois, prendront des hormones pour les aider à développer, biologiquement parlant, un certain nombre de traits physiques secondaires liés au genre auquel elles s'identifient?

L'expression de genre, pour sa part, est généralement abordée sous l'angle de la sociologie et de l'anthropologie. Elle réfère à la façon dont la personne exprime son identité de genre ${ }^{8}$. Habituellement, les hommes "chercheront» à mettre en évidence certains traits de leur masculinité et ils tâcheront de « cacher » ceux qui pourraient les faire paraitre moins conformes aux stéréotypes de genre. De nombreuses femmes feront aussi tout ce qui est en leur pouvoir pour mettre en évidence leur féminité, mais, sous l'influence du mouvement fémi- niste et de la mode occidentale du XXe siècle, certaines pourront aussi se vêtir de manière beaucoup plus masculine que ce que les stéréotypes de genre leur « recommanderaient». Entre ces deux extrémités du continuum, une immense variété existe encore une fois. Alors que certaines personnes chercheront sur une base permanente à adopter un genre «passe-partout» (aussi parfois appelé « look androgyne » ou personne «agenre »), d'autres exprimeront «à temps plein» un genre en plus ou moins grande opposition à leur sexe biologique. Nous pourrons alors parler d'hommes efféminés, de « gars manqués » ou de « filles manquées » (selon des expressions québécoises péjoratives bien connues). Enfin, il faut aussi savoir que cette expression de genre distincte du sexe biologique peut être exprimée à temps partiel. Selon les motifs invoqués par les personnes pour porter des vêtements traditionnellement associés à l'autre genre que celui qui leur a été assigné à la naissance, nous pourrons alors parler des personnes travesties (où le confort et le plaisir comptent parmi les motifs évoqués) ou des personnes «drag» (où le port des vêtements du genre opposé au sexe biologique sert à des fins de spectacles le plus souvent humoristiques ${ }^{9}$ ). La personne travestie, contrairement à la personne transgenre et à la personne transsexuelle, ne s'identifie donc pas à un genre différent de celui qui lui a été assigné à la naissance. Ce ne sont que les apparences (dont les vêtements) de «l'autre genre » qui l'intéressent. Pour faire un lien avec ce qu'en dit la psychiatrie, la personne travestie ne souffre donc pas de dysphorie de genre ou de trouble de l'identité de genre.

Le quatrième repère qu'il importe de considérer pour bien comprendre notre définition de ce qu'est une personne trans*, c'est celui de l'orientation sexuelle. À chacune des extrémités de ce repère, on retrouve la même notion : l'hétérosexualité qui réfère au fait qu'une personne est sexuellement attirée par une personne dont le sexe biologique est opposé au sien. Entre ces deux extrêmes, toutefois, nous retrouvons encore une fois une très grande variété de nuances dont la plus connue est bien entendu l'homosexualité (soit l'attirance pour une personne du même sexe biologique que le sien). Si une personne est attirée à la fois par les personnes de l'autre sexe biologique et du même sexe biologique que le sien, nous dirons qu'elle est bisexuelle. Si une personne est quant à elle attirée par des gens 
se situant n'importe où sur ce continuum nous dirons qu'elle est pansexuelle. À l'inverse, si l'individu en question n'éprouve aucune attirance sexuelle, nous dirons alors qu'il est asexuel ${ }^{10}$.

Depuis une vingtaine d'années, un mouvement social visant à remettre en cause ces quatre catégories binaires inégalitaires historiquement et géographiquement situées ${ }^{11}$ a vu le jour. C'est le mouvement queer (Bourcier, 2006; Hall, 2013; Navarro Swain, 1998; Wilchins, 2004). Selon cette théorie (que certains considèrent comme portée par des personnes «freaks » issues tout droit de certains campus universitaires américains ${ }^{12}$ ), il ne faut pas seulement reconnaitre aux homosexuels les mêmes droits qu'aux personnes hétérosexuelles. Il faut reconnaître, à tout individu, le droit d'être reconnu comme un être humain à part entière, peu importe son sexe biologique, son identité de genre, son expression de genre et son orientation sexuelle. En fait, pour le mouvement queer, c'est le système binaire présent dans la société qu'il faut remettre en cause, car les corps ont tendance à y être considérés comme des surfaces vierges où viennent s'y inscrire les rapports de pouvoir inégalitaires entre les groupes sociaux (Davy, 2011) ${ }^{13}$.

\section{Pourquoi ne voit-on pas davantage de personnes trans* participer au marché du travail}

Divers écrits sociologiques (voir, par exemple, Sanger, 2010) et anthropologiques de la même trempe que celui de Butler (1990) nous aident à comprendre plus facilement pourquoi on ne voit pas davantage de personnes trans* participer au marché du travail. Selon cette perspective, les deux extrémités du modèle binaire n'ont pas la même valeur ni la même désirabilité sociale (Maillé, 2012). Dit autrement, l'Occident serait depuis de nombreux siècles une société patriarcale dominée par des hommes hétérosexuels (The One.) où les femmes (The Other:) n'ont « rien » en elles-mêmes... sinon que d'être l'Autre... la personne qui n'a pas «le pénis », "l'intelligence», «la raison», voire «le droit et la capacité d'être entièrement libre et indépendante » (Larivière, 2017) ${ }^{14}$. Derrière ces diverses inégalités «[...] se cachent des rapports de pouvoir, des gagnants et des perdants, des hommes et des femmes qui ont des choses à gagner si les règles du jeu changent, mais aussi parfois des choses à perdre (intérêts financiers, politiques et symboliques). Cela pourrait expliquer les résistances et les blocages » (Cornet, 2014, p. 66).

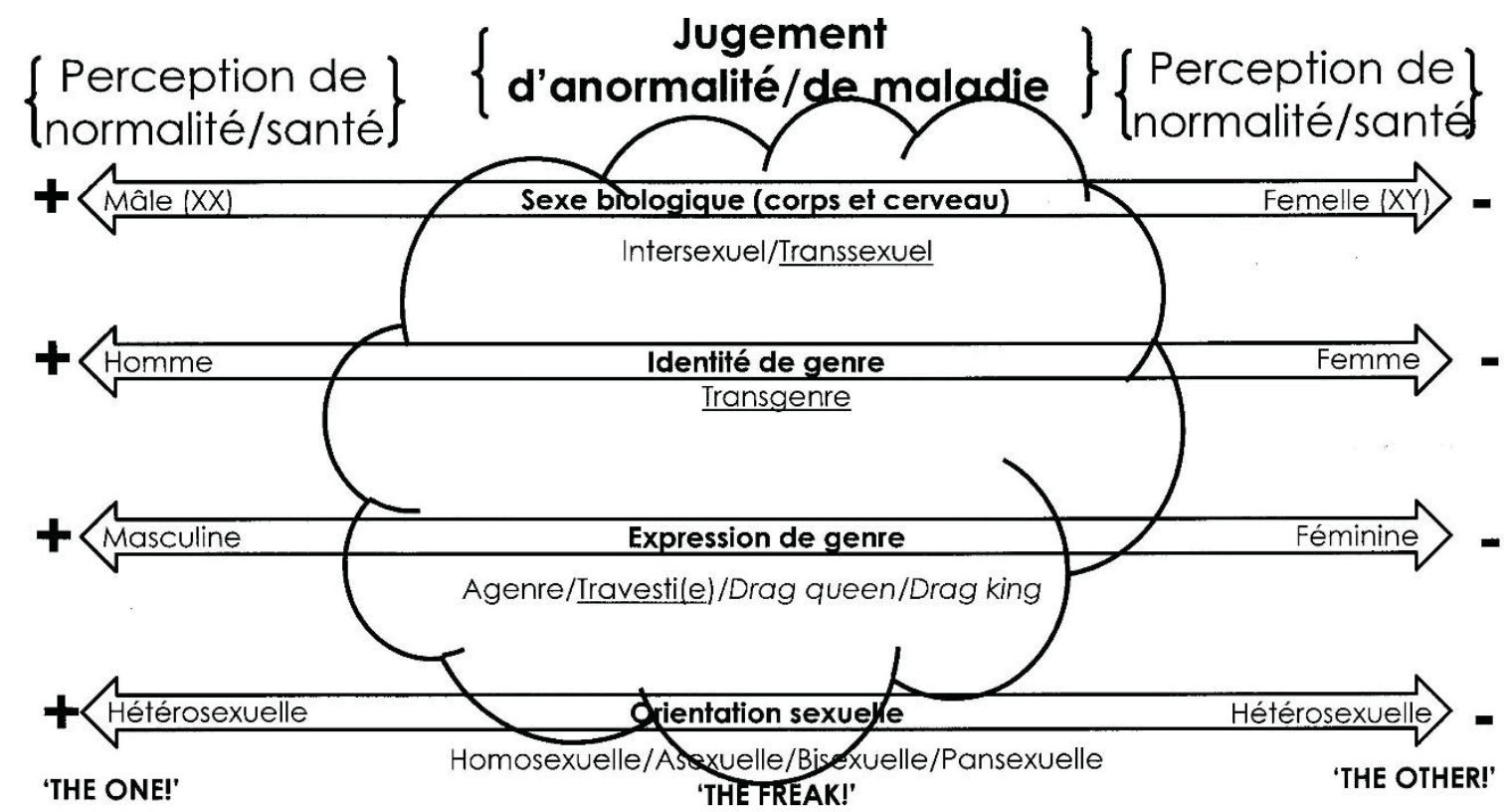

Figure 1 - Repère fondamentaux pour comprendre la réalité des personnes trans 
Ce que cette vision de la société nous permet d'entrevoir au sujet des personnes trans* c'est que les hommes trans* (c'est-à-dire les hommes nés dans un corps de femme) seraient potentiellement l'objet de beaucoup moins de railleries et de discrimination que les femmes trans* (c'est-à-dire les femmes nées dans un corps d'homme). En d'autres mots, nous pouvons faire l'hypothèse que la société patriarcale, par l'entremise de ses membres, comprend et accepte beaucoup plus facilement qu'une femme s'habille en homme pour obtenir des privilèges, qu'elle n'aurait pas autrement, qu'un homme s'habille en femme pour renoncer aux privilèges auquel il a déjà droit... surtout si la transition d'un genre à l'autre est encore très « visible » (Mattilda, 2006). Alors que la première personne est plus facilement comprise et jugée " intelligente », l'autre serait davantage incomprise, rejetée et considérée comme folle ${ }^{15}$.

L'étude de la question des personnes trans* sur le marché du travail ne saurait être complète sans la prise en compte de ce que le sociologue JeanFrançois Amadieu appelle «le poids des apparences » et de la beauté (Amadieu, 2002). Dans l'un de ses ouvrages les plus importants, intitulé La société du paraître - Les beaux, les jeunes... et les autres
(2016), il démontre à quel point, notamment sur le marché du travail, nos sociétés sont généralement empreintes d'attentes très élevées à l'égard des apparences des gens et elles contribuent, de ce fait, à créer une véritable armée des exclus du paraitre. Les individus, nous explique-t-il, sont systématiquement évalués (parfois même de manière inconsciente) au regard des « canons » de beauté, puis des décisions d'embauche (notamment) sont prises sur ces apparences. Plus un individu répond positivement aux canons de la beauté de la société dans laquelle il évolue, plus sa candidature est jugée «bonne» et en adéquation avec les compétences recherchées. C'est ainsi, ne manque-t-il pas de nous expliquer, que dans plusieurs pays occidentaux, à compétences égales, les beaux et les jeunes venant d'une «bonne famille» sont systématiquement favorisés au détriment des autres (Amadieu, 2006). Loin d'être en régression, ce phénomène serait même l'une des composantes principales de l'hypermodernité libérale (Lipovetsky, 2017). Bref, pour décrocher un emploi et le conserver lorsque les conditions économiques deviennent plus difficiles, il faut répondre aux canons de la beauté au sein de notre société. Autrement, nous en serons les premiers sacrifiés.

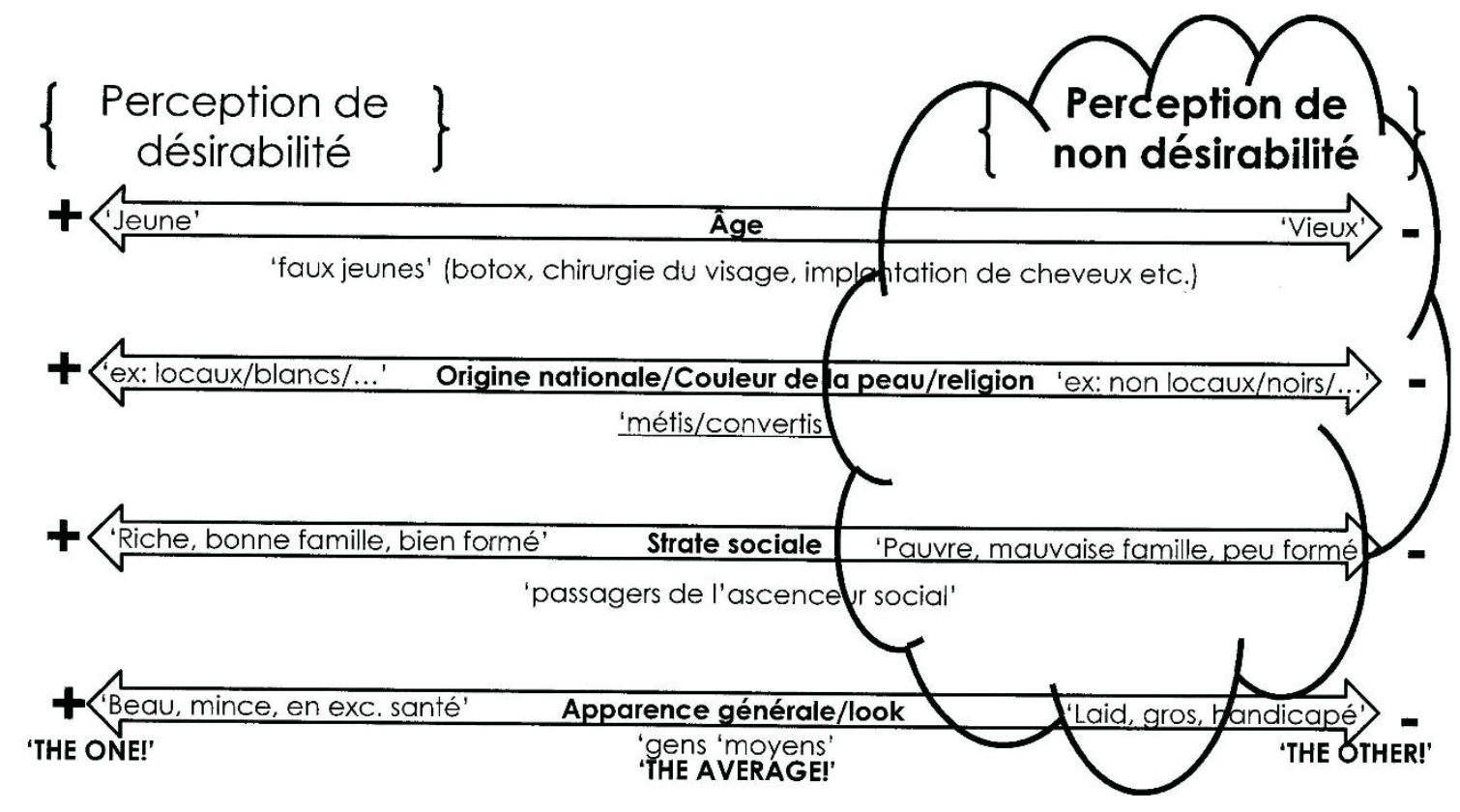

Figure 2-Repères supplémentaires pour comprendre la réalité des personnes trans 
Si nous croisons les deux tableaux précédents, nous pouvons plus aisément comprendre les difficultés extrêmes vécues par certaines personnes trans* sur le marché du travail et pourquoi, dans les faits, elles y sont si peu présentes. Bien qu'aucune donnée empirique québécoise et solide n'ait jamais été collectée sur cette question, nous pouvons faire l'hypothèse que toutes les personnes trans* ne seront pas victimes d'autant de violences et de discrimination. Théoriquement du moins, nous pouvons présumer que ce sont surtout celles qui ne réussissent pas à " passer incognito », pour de jeunes, belles et riches personnes du genre auquel elles s'identifient, qui rencontreront vraisemblablement le plus d'obstacles et se heurteront à plus de rejet. Cela sera d'autant plus le cas que ces personnes trans* cumulent, en plus, la présence d'un handicap physique, ou le manque de maitrise de la langue parlée qui s'explique, notamment, par une origine nationale autre reconnaissable aussi par la couleur de la peau ${ }^{16}$.

Au sexisme rampant, il semblerait que l'on peut donc ajouter l'âgisme, le racisme, l'homophobie et la transphobie. Si la question du racisme est déjà connue et que l'on sait à quel point les problèmes vécus aux Etats-Unis par les personnes trans* d'origine sudaméricaine ou africaine sont infiniment plus grands que les problèmes vécus par les personnes trans* dites «blanches ${ }^{17}$, la transphobie mérite qu'on s'y attarde quelques instants : c'est la peur irrationnelle et l'hostilité démontrée à l'égard des personnes trans* (Harrington, 2016) et qui fait en sorte, au cours des processus d'embauche, que les candidatures des personnes trans* sont instantanément rejetées. Comme de nombreuses autres peurs et manifestations de rejet, elles prennent racine, entre autres choses, dans l'ignorance qui alimente, à son tour, stéréotypes et préjugés (Calvez, 2009). Bien qu'aucune recherche québécoise n'ait encore été faite pour valider les hypothèses émises dans cet article, la littérature nous permet d'avancer que si l'on ne voit pas davantage de personnes trans* participer au marché du travail, c'est pour deux raisons : certaines répondent si bien aux " canons » de la beauté du genre auquel elles s'identifient qu'elles sont embauchées, mais que l'on ne sait tout simplement pas que ce sont des personnes trans*; d'autres, toutefois, répondent si mal à ces mêmes canons qu'elles sont automatiquement rejetées des processus de sélection du personnel et, lorsque les affaires commencent à moins bien aller, elles sont les premières à être licenciées.

\section{Les personnes trans* selon certaines figures d'autorité}

\author{
"To define is to limit $[\ldots]$ \\ how shallow were the arbitrary definitions \\ of ordinary psychologists 》 \\ - Oscar Wilde
}

La chrétienté a marqué depuis de nombreux siècles, et continue de marquer, de nombreuses sociétés. Pour certains fidèles, basant leurs prises de position sur la Bible, les personnes trans* ne sont pas des personnes parfaitement saines de corps et d'esprit. Ce sont, plutôt, des pécheurs et des déviants sexuels en puissance qui posent des gestes contraires aux lois divines. Cette attitude négative à propos des personnes trans* part de l'idée que Dieu aurait fait l'Homme à son image et que l'on ne pourrait donc pas modifier notre corps et son apparence; cela reviendrait à altérer l'image de Dieu. Nous ne pourrions pas, non plus, aimer une personne du même genre que soi, car ce serait céder aux plaisirs de la chair sans aucune chance de donner la vie. Dans l'ouvrage principal que ces adhérents utilisent pour appuyer leurs propos, notre retrouvons le passage suivant tiré de l'Ancien Testament (Deutéronome 22-5) : «Une femme ne portera point un habillement d'homme, et un homme ne mettra point des vêtements de femme; car quiconque fait ces choses est en abomination à l'Éternel, ton Dieu » (repéré à https://doublegenre.wordpress.com/2016/04/30/a-tous-lestransphobes-citant-la-bible/). Au sujet spécifique de l'homosexualité, bien que cette notion n'entretienne aucune relation avec le fait d'être une personne trans*, comme nous le disions, le passage suivant tiré également de l'Ancien Testament (Lévitique 20,13) est très clair: "Quand un homme couche avec un homme comme on couche avec une femme, tous deux commettent une abomination; ils seront mis à mort, leur sang retombera sur eux» (repéré à https://www.devenirunenchrist.net/chretiens-et-homosexuels/que-disentla-bible-et-leglise-sur-lhomosexualite.html). Bien que ces deux parties des Écritures n'aient pas été reprises comme telles dans le Nouveau Testament, nul besoin de chercher très loin pour trouver des figures d'autorité religieuse qui partagent, encore de nos jours, cette opinion pourtant ancienne selon laquelle les personnes trans* sont des gens « contre nature » qui ne respectent pas la loi de Dieu. En conséquence, selon cette vision, la société n'a pas à 
protéger les personnes trans*. Au contraire, elle doit même se protéger d'elles! C'est ainsi qu'à certaines époques, on a cru qu'on devait mettre à mort de telles personnes ${ }^{18}$.

Fort heureusement, on sait aujourd'hui qu'il y a aussi dans la chrétienté des prêtres et des pasteurs qui ont une vision beaucoup moins négative à l'égard des personnes trans* et des personnes homosexuelles (Yarhouse, 2015). Également, grâce en partie au mouvement féministe, si les femmes sont davantage acceptées lorsqu'elles portent des habits traditionnellement associés à la gent masculine, il n'en reste pas moins que dans la plupart des sociétés occidentales les hommes qui portent des habits féminins continuent de ne pas être considérés avec autant de sérieux et de respect.

Pour certains membres du corps médical, encore de nos jours, les personnes trans* souffrent d'un trouble mental répertorié dans le $D S M V^{19}$ et qui, s'il n'est pas traité, peut les conduire au suicide (Becker et Perkins, 2014; Desjardins, 2013; Lawrence, 2014) ${ }^{20}$. Élaboré pour la première fois en 1952 par la Société américaine de psychiatrie, cet ouvrage de référence a vu sa cinquième édition être lancée en 2013 sans avoir pu faire l'économie d'une certaine critique, même à l'intérieur du cercle restreint des psychiatres (Corcos, 2015) ${ }^{21}$. À l'intérieur de ce document qui fait autorité parmi les membres de la communauté médicale, la question est abordée sous l'angle de la notion de dysphorie de genre que l'on définit de la manière suivante: "Incongruence marquée entre le genre expérimenté/ exprimé par un individu et son genre assigné (à la naissance) » [notre traduction] (DSM-5 Diagnostic Criteria for Gender Dysphoria in Adolescents and Adults/302.85 [F64.1]) $)^{22}$.

Pour être avéré, ce diagnostic repose sur quatre critères principaux: "une identification intense et persistante à l'autre sexe, un sentiment persistant d'inconfort par rapport au sexe assigné, l'absence d'affection intersexuelle physique et une "souffrance cliniquement significative" " (Steinberg, 2013, p. 164). Pour corriger la dysphorie de genre, diverses approches ont été tentées au fil du temps. Alors que de la fin du XIX ${ }^{\mathrm{e}}$ siècle jusqu'au milieu du XX $\mathrm{XX}^{\mathrm{e}}$ siècle certains médecins ont tenté de soulager les personnes trans* en leur administrant des électrochocs «réparateurs» accompagnés de séances de psychanalyse de reconversion visant à leur « faire comprendre » qu'elles étaient malades et qu'il fallait qu'elles retrouvent la raison ${ }^{23}$, les traitements «modernes» consistent à permettre aux personnes trans* (sous certaines conditions) de recevoir, entre autres choses, des interventions chirurgicales et des traitements hormonaux en lien avec le genre auquel elles s'identifient ${ }^{24}$. Il faut enfin savoir que les recherches empiriques menées sur l'efficacité de ces divers plans de traitement ont souligné très clairement l'absence d'effets positifs des premiers modes de traitement (voire leurs effets très négatifs) et, globalement, les effets positifs des seconds (Pfafflin et Junge, 1998).

Pour certaines féministes radicales, dont Janice Raymond, les femmes trans* ne sont rien d'autre que le produit de la société machiste patriarcale contemporaine et d'un « empire transsexuel » qu'il faut combattre, car il divise les femmes en cherchant à faire "pénétrer dans leurs espaces » des hommes qui ne devraient pas y être. Si, en tant qu'individus, les personnes trans* ne méritent pas d'être victimes de violence, il ne faut pas pour autant les croire lorsqu'elles affirment qu'elles sont des femmes, car elles ne le sont pas.... Elles se trompent... elles ne peuvent pas être ce qu'elles disent qu'elles sont. Les croire revient à les encourager dans leurs erreurs. En conséquence, Raymond croit qu'il ne faut surtout pas autoriser ce qu'elle appelle les chirurgies non nécessaires et les mutilations médicales transformant des hommes en femmes. Il faudrait plutôt, à son avis, éliminer le système social et médical qui soutient le phénomène transsexuel et renforce, plus globalement, la domination des femmes par les hommes et les raisons pour lesquelles certaines personnes considèrent nécessaire de changer de corps.

Dans son ouvrage phare intitule The Transsexual Empire (Raymond, 1979), elle avance l'idée que les femmes trans* sont des hommes manipulés et fabriqués par d'autres hommes rendus capables, grâce à l'évolution de la médecine, de transformer « en apparences », et selon l'image qu'ils en veulent, des hommes en femmes. En ce sens, les transsexuelles ne sont que des produits machistes servant à renforcer les stéréotypes de genre traditionnels et la société patriarcale dans son ensemble. L'un des passages les plus tristement célèbres de son ouvrage est le suivant : 
Toutes les transsexuelles violent les corps des femmes en réduisant la forme réelle de la femme à un artéfact pour ensuite s'en approprier le corps pour elles-mêmes. Cela étant dit [il faut savoir que] les féministes lesbiennes construites de manière transsexuelle violent en plus la sexualité des femmes et leur esprit. [Sous-entendant que plusieurs femmes trans* cachent aux autres le fait qu'elles soient nées dans un corps d'homme elle poursuit en écrivant] : Le viol, bien qu'il soit généralement fait par la force, peut aussi être accompli par la duperie [notre traduction] (Raymond, 1979, p. 104).

Considérée aujourd'hui par certains comme l'une des personnes les plus transphobes n'ayant jamais écrit sur le sujet, on ne peut nier que Raymond propose une réflexion « originale et critique». Toutefois, il faut préciser que ses prises de position sont ouvertement réprouvées et remises en question par certaines féministes « libérales » n'ayant pas du tout les mêmes vues sur le phénomène transsexuel (Tosh, 2016) ${ }^{25}$. Si la thèse de Raymond rejoint en partie certains auteurs très critiques de notre société pour qui le genre y est trop normé, stéréotypé et injuste envers les femmes, son propos manque clairement de nuances et néglige complètement la prise en compte de la réalité des hommes trans ${ }^{* 26}$.

Enfin, ce rapide tour d'horizon de ce que sont les personnes trans* selon certaines «figures d'autorité » ne saurait être complet sans un examen de la contribution mise de l'avant par certains historiens et anthropologues qui soutiennent que les personnes trans* sont tout simplement des personnes comme les autres faisant partie d'une autre catégorie sociale que celles auxquelles on fait habituellement référence en Occident. Par contre, ce n'est que depuis les années 1990 que le mot trans* serait utilisé de manière populaire, essentiellement en Occident (Ekins et King, 2006). Auparavant, on appelait les personnes trans* "personnes homosexuelles » ou «personnes travesties » (Valentine, 2007). De surcroit, les personnes trans* auraient non seulement existé de tout temps, mais, aussi dans toutes les sociétés (Goguel d'Allondans, 2017 ${ }^{27}$. Selon certains activistes et chercheurs, il existerait certaines sociétés où, encore de nos jours, les personnes trans* sont l'objet d'une certaine vénération de la part des membres de leur communauté (Feinberg, 1996; Totman, 2003) et d'autres où l'on assisterait, depuis les années 1960, au développement en puissance d'un véritable mouvement «trans» chamboulant au passage le mouvement féministe « classique » (Stryker, 2008). Bref, de ce point de vue, il est impossible de nier l'existence des personnes trans* et il n'y a donc pas lieu de « corriger » la problématique en usant de répression à l'endroit des personnes trans* ou d'un quelconque « empire transsexuel », le phénomène étant tout à fait naturel et universel. Selon cette perspective, que l'on considère comme étant la plus raisonnable de toutes celles présentées jusqu'ici, les personnes trans* ne représentent qu'une variante parmi d'autres de l'espèce humaine ${ }^{28}$.

\section{Les droits des personnes trans*}

Ce n'est pas le cœur de notre propos que de présenter les différentes dispositions légales protégeant, au Québec, les droits des personnes trans*. Il nous suffit toutefois de dire que ce n'est que depuis le mois de juin 2016 que l'identité de genre et l'expression de genre font partie des motifs illicites de discrimination prévus à l'article 10 de la Charte québécoise des droits et libertés de la personne. Auparavant, la discrimination à l'égard des personnes trans* était implicitement interdite en raison de l'interdiction de discrimination sur la base du sexe (Sauvé, 2015) et du handicap. Ce qu'il importe de souligner, à notre avis, c'est que très peu de sociétés offrent aujourd'hui des protections explicites à l'égard de leurs citoyens dont l'identité de genre et l'expression de genre diffèrent du sexe qui leur a été assigné à la naissance (Gouvernement du Québec, 2014). Qui plus est, à l'heure où ces lignes sont écrites, l'homosexualité, à laquelle on associe encore trop souvent les personnes trans*, est encore passible de la peine de mort dans plus d'une demi-douzaine de pays (dont l'Iran) (repéré à https://lesobservateurs.ch/2017/04/04/rappelsur-les-7-pays-qui-condamnent-toujours-les-homosexuels-a-la-peine-de-mort-tous-sont-musulmans-silence-des-associations/).

\section{Les violences dont sont victimes les personnes trans*}

Les inégalités vécues par les personnes trans* sur le marché du travail trouvent leur origine, au moins en partie, dans certaines des conceptions péjoratives présentées précédemment (notamment celles 
véhiculées par certaines autorités religieuses, médicales et féministes) et l'absence de dispositions législatives protégeant leurs droits. Se conjuguant les uns aux autres, ces éléments contribuent à générer au sein de la population une croyance selon laquelle les personnes trans* seraient indésirables à cause de leurs gestes et comportements et n'auraient pas, en conséquence, les mêmes droits que les gens «normaux ». Répétées depuis des siècles et prononcées encore aujourd'hui à satiété sur certaines tribunes réputées pour leurs propos éminemment « conservateurs », ces « convictions » contribuent à générer de multiples microagressions et actes de discrimination à l'encontre des personnes trans* et à nuire à la pleine reconnaissance de leurs droits (Nadal, 2013) ${ }^{29}$. Le tableau suivant, loin d'être exhaustif, présente quelques exemples de ces formes de violence dont sont victimes les personnes trans*.

\begin{tabular}{|l|l|l|}
\hline Violences insidieuses & Violences graves & Violences extrêmes \\
\hline Ignorance « crasse » & Intimidation & Voies de fait \\
\hline $\begin{array}{l}\text { Regards et gestes } \\
\text { méprisants }\end{array}$ & $\begin{array}{l}\text { Harcèlement } \\
\text { psychologique }\end{array}$ & Agression sexuelle \\
\hline Médisance & Harcèlement sexuel & Menaces de mort \\
\hline Insultes & $\begin{array}{l}\text { Menaces de voies de } \\
\text { fait }\end{array}$ & Torture \\
\hline $\begin{array}{l}\text { Rémunération } \\
\text { injuste }\end{array}$ & $\begin{array}{l}\text { Utilisation } \\
\text { consciente et répétée } \\
\text { du mauvais nom, } \\
\text { prénom ou genre }\end{array}$ & Meurtre \\
\hline $\begin{array}{l}\text { Mutation à des } \\
\text { horaires de travail } \\
\text { indésirables }\end{array}$ & $\begin{array}{l}\text { Discrimination à } \\
\text { l'embauche }\end{array}$ & Emprisonnement \\
\hline $\begin{array}{l}\text { Mutation à des postes } \\
\text { de travail } \\
\text { indésirables }\end{array}$ & $\begin{array}{l}\text { Refus de rendre } \\
\text { service }\end{array}$ & $\begin{array}{l}\text { Institutionnalisation } \\
\text { psychiatrique } \\
\text { (traitements forcés) }\end{array}$ \\
\hline $\begin{array}{l}\text { Refus de collaborer } \\
\text { Refus de donner des }\end{array}$ & Automutilation*30 \\
\hline $\begin{array}{l}\text { Utilisation consciente } \\
\text { du mauvais nom, } \\
\text { prénom ou genre }\end{array}$ & Congédiement & Suicide* \\
\hline \multicolumn{2}{|l|}{} & \multicolumn{2}{|l|}{}
\end{tabular}

Tableau 1 - Quelques formes de violence dont sont victimes les personnes trans*

Diverses études empiriques récentes menées auprès de jeunes personnes LGBTQ ont démontré que ces actes de violence ont tendance à s'enchaîner et à s'additionner les uns aux autres pour créer une sorte de spirale autodestructive où la victime commence par se retirer (ou est carrément éjectée) de son milieu de vie habituel (famille, école et emploi) pour éventuellement se résoudre à vivre sans emploi ou d'emplois illicites (notamment dans l'univers de la prostitution) (Dorais, 2014). Il semblerait, enfin, qu'une proportion importante de ces jeunes personnes trans* finit littéralement « dans la rue » en venant grossir les rangs des personnes itinérantes quand ce n'est pas en établissement carcéral ou psychiatrique ou encore à la morgue, des suites d'un suicide (Meyer, 2015). Claude Charron, dans l'ouvrage de Pellan (2005, p. 10), écrit au sujet de cette spirale autodestructive vue de l'intérieur:
L'homosexuel découvre l'homophobie avant même qu'il ne reconnaisse sa différence. Si ce n'est pas dans sa famille, ce sera très tôt à l'école ou dans la rue. Il saura qu'on l'exclut pour ce qu'il est, même en devenir. La douleur vient de l'extérieur, mais une fois intériorisée, sa transformation en haine de soi-même peut devenir intolérable. Certains craquent sous le supplice infligé à l'âme [...]. Quand un homosexuel meurt, il ressemble, bien sûr, à tout humain qui meurt. Mais sa mort porte aussi une différence. Il ne sait, pas plus à cet instant qu'à tous les autres de sa vie, pourquoi il a été différent des autres et pourquoi on avait peur de lui. Depuis des siècles et pour des siècles. 
Parlant aussi de cette spirale de violence, mais en des termes moins personnels, Taubira écrit : «Lorsque commence ce jeu pervers et nauséabond de rejet de l'autre, de stigmatisation de la différence, cela commence par les différences visibles et finit par les différences imaginaires; cela commence par les ricanements, cela finit par des meurtres isolés ou en série (Taubira, 2014, p. 130). Afin d'aider individuellement les personnes trans* à mieux faire face à toutes ces formes de violence dont elles sont l'objet, certains auteurs (dont Singh, 2018) ont commencé à publier des manuels afin de les aider à développer leurs propres capacités de résilience ${ }^{31}$.

\section{La gestion du personnel trans* sur le marché du travail}

La littérature managériale s’intéresse depuis déjà quelques années à la diversité de la main-d'œuvre età ses impacts généralement positifs sur le fonctionnement organisationnel (Chicha et Saba, 2010; Page, 2017; Peretti, 2012a; Peretti, 2012b). Cela étant dit, rares sont les recherches qui ont porté sur la diversité portée par les personnes trans* (Malo, 2018). Le peu que l'on sait est d'ailleurs très préoccupant comme l'écrivaient encore récemment deux expertes américaines :

As they become adults, (american) trans people face barriers at every level when it comes to employment. They are less likely to have family support and access to higher education, less likely to be hired and more likely to be let go. Their unemployment rate is twice the national average and for the black trans people, four times the average. (Erickson-Schroth et Jacobs, 2017, p. 107)

Cet extrait confirme l'idée selon laquelle les personnes trans* ne sont pas que victimes d'incompréhension conduisant à toutes sortes d'actes de violence (allant de la microagression de type «insulte voilée» au meurtre [Teitel, 2015], mais aussi de discrimination à l'embauche et au congédiement sans cause juste et suffisante) (Bender-Baird, 2011; Gibney, 2016; Grant et al., 2011; Klie, 2009; Trans Pulse, 2011).

Une enquête récente menée aux États-Unis auprès de la population trans* a révélé que $90 \%$ des répondants ont déjà été harcelés ou maltraités dans le cadre de leur emploi sur la base qu'ils étaient trans*. Au sein du même échantillon, $26 \%$ des répondants ont dit avoir déjà perdu un emploi à cause du fait qu'ils étaient trans* (Tuttle, 2015). Au Québec, selon l'organisation Aide aux trans du Québec, entre $13 \%$ et $56 \%$ des personnes trans* ayant répondu à une enquête ont affirmé avoir perdu leur emploi pour des raisons d'identité de genre et entre $13 \%$ et $47 \%$ se sont vu refuser un emploi pour des raisons d'identité de genre (ATQ, 2016) ${ }^{32}$. Enfin, selon le projet de recherche canadien Trans Pulse (cité par le Conseil québécois LGBT, 2017), 44 \% des personnes trans* identitaires auraient un diplôme postsecondaire, collégial ou universitaire, mais leur revenu annuel est inférieur à 15000 \$. Le reste de la population trans* serait quant à elle bénéficiaire de l'aide sociale, de l'assurance-emploi ou du chômage, selon les programmes gouvernementaux offerts dans chaque province. Dans un contexte économique québécois marqué de plus en plus par des pénuries de main-d'œuvre et où un nombre sans cesse croissant d'organisations disent éprouver beaucoup de difficultés à pourvoir les emplois vacants, n’est-il pas étrange que les compétences professionnelles des personnes trans* ne soient pas davantage reconnues?

Maintenant que nous comprenons davantage ce que sont les personnes trans*, les violences dont elles sont victimes et les raisons pour lesquelles cette animosité a tendance à perdurer, le moment est venu de nous pencher sur les solutions que l'on pourrait mettre de l'avant afin de les protéger davantage et mieux les intégrer au marché du travail.

\section{Les solutions pour aider les personnes trans* à s'intégrer au marché du travail}

"Les droits de toutes les personnes sont diminués lorsque les droits de l'une d'entre elles sont menacés» - John Fitzgerald Kennedy

De nombreuses choses peuvent, et devraient, être faites sur le marché du travail pour aider les personnes trans*. Aucune, toutefois, n'est du ressort exclusif d'un seul acteur ou d'une seule catégorie d'acteurs. Selon nous, les trois acteurs principaux du système des relations industrielles (soit l'État, les employeurs et les syndicats) doivent travailler en étroite collaboration. Examinons, sans plus tarder, certaines des actions que ces trois grands acteurs peuvent, de concert, mettre de l'avant. 


\subsection{Responsabilités de l'État}

Comme les recherches anthropologiques nous l'ont bien démontré, les personnes trans* ont de tout temps existé, et cela, à toutes les époques. Nous pouvons donc en conclure qu'elles sont, à cet égard, des citoyennes comme les autres et qui méritent, comme les autres, de voir reconnaitre leurs droits à vivre sereinement sans faire l'objet de violences de toutes sortes. Cela implique, à notre avis, que la première responsabilité des États est d'interdire explicitement toute forme de discrimination sur la base de l'identité de genre, l'expression de genre et les apparences ${ }^{33}$ (particulièrement sur le marché du travail).

En complément avec cette première piste de solution, trois autres choses peuvent aussi être faites par les pouvoirs publics pour protéger et promouvoir les droits des personnes trans*.

Premièrement, nous croyons que toute personne trans* qui le souhaite devrait pouvoir changer officiellement dans son acte de naissance, son passeport et tous les documents la concernant, son nom et son prénom, de même que la photo qui y figure, de telle sorte qu'ils soient conformes à son identité et à son expression de genre ${ }^{34}$.

Deuxièmement, nous croyons que toute personne trans* qui le souhaite devrait pouvoir changer officiellement dans son acte de naissance, son passeport et tous les documents la concernant la mention de son genre, de même que la photo qui y figure, de telle sorte qu'elles soient conformes à son identité et à son expression de genre ${ }^{35}$. Considérant, sous l'impulsion du mouvement queer, que certaines personnes pourraient même ne pas se sentir à l'aise de s'identifier à l'un ou l'autre des genres «habituels ", l'État devrait aussi songer à permettre l'existence, dans ses divers documents administratifs, d'autres catégories (par exemple : Autre; Trans*; Non identifié; Confidentiel; etc.) voire ne plus les utiliser du tout (Davis, 2017).

Troisièmement, nous croyons que toute personne trans* qui le souhaite devrait pouvoir bénéficier, gratuitement, de tous les services nécessaires pour l'aider à vivre sa transition dans les meilleures conditions possibles. Cela implique, pour celles qui le désirent, de pouvoir bénéficier de soutien psychologique, de pouvoir recevoir des hormones et d'avoir accès à toutes les interventions chirurgicales et esthétiques nécessaires pour l'aider à vivre dans le genre auquel elle s’identifie (cela inclut, sans s'y limiter, les opérations de réassignation sexuelle [vaginoplastie; phalloplastie; etc.]; les interventions visant la pose d'implants mammaires et la mastectomie; la suppression de la pomme d'Adam; les chirurgies de féminisation du visage; les services d'électrolyse; etc. ${ }^{36}$.

Du même coup, il nous semble important que les États rendent possible ces diverses choses sans pour autant demander, aux personnes trans*, des conditions trop sévères qui auraient pour effet de restreindre leurs droits à disposer de leur propre corps, par exemple, la présentation d'un nombre élevé de lettres de spécialistes de la santé confirmant que la personne en question souffre d'un trouble mental ou, encore, qu'elle a subi avec succès une opération de réassignation sexuelle l'ayant rendue stérile. Dit autrement, nous sommes d'avis qu'il ne faut pas que les États forcent les personnes intersexuelles, transgenres ou travesties à devenir transsexuelles.

Enfin, une fois mises en place toutes ces mesures, il va sans dire qu'il est aussi de la responsabilité des États de les faire connaitre et s'assurer qu'elles soient effectivement respectées par toutes les personnes concernées. Autrement, bien qu'elles aient l'égalité juridique (ou de droit), rien ne pourra assurer aux personnes trans* que cette dernière se matérialise concrètement en égalité sociale (ou de fait) (Bourré, 2017) ${ }^{37}$.

\subsection{Responsabilités des employeurs ${ }^{38}$}

Les employeurs occupent une place privilégiée dans la société et ce sont souvent eux, sur le marché du travail, qui sont aux premières loges pour témoigner et éventuellement contrer les divers phénomènes de violence dont les personnes trans* sont souvent l'objet. Ils peuvent donc, à notre avis, faire diverses choses pour aider les personnes trans* à vivre normalement.

Tout d'abord, il nous semble fondamental que tous les employeurs adoptent un code de conduite à l'intention de tous leurs employés (incluant leurs gestionnaires) qui stipule que l'organisation ne démontrera aucune tolérance pour tout acte de violence (microagressions et autres) commis à l'égard d'une personne trans* (que cette dernière soit une employée de l'organisation, une cliente, une fournisseuse de services, une sous-traitante ou autre). Comme les collectifs de travail sont parfois le siège de nombreuses microviolences à l'égard des personnes trans*, les employeurs ont la responsabilité 
de s'assurer que de tels comportements soient clairement reconnus comme étant inacceptables et passibles de sanctions disciplinaires.

Deuxièmement, en relation avec la clientèle, les employeurs ont aussi une certaine responsabilité. Sans pouvoir "contrôler» les comportements de leurs clients, ils n'en ont pas moins la possibilité de les influencer de manière positive. À titre d'exemple, si un client menace une entreprise de ne plus faire des affaires avec elle parce qu'elle emploie une personne trans*, l'employeur peut lui expliquer à quel point il est important de respecter tous les gens, peu importe leur identité et leur expression de genre. À la fin, si le client décide de cesser ses relations d'affaires avec l'entreprise, il ne pourra bien entendu rien y faire. Pour contrer tout de même une telle éventualité, l'employeur pourra prendre les devants et annoncer son intention de développer sa capacité à valoriser toutes les diversités de la main-d'œuvre et, ainsi, préparer psychologiquement ses clients à rencontrer ses propres employés. Cela ne pourra, à notre avis, que l'aider à pourvoir plus facilement ses postes, mais, aussi, à attirer de nouveaux clients eux-mêmes ouverts à cette diversité. Dans un contexte de pénurie de main-d'œuvre, cela nous semble être une piste très intéressante à explorer.

Troisièmement, lorsque les employeurs comptent dans leurs rangs une personne trans* qui leur annonce son intention de faire sa transition d'un genre à l'autre (ou de se vêtir de temps à autre) dans un genre qui n'est pas celui qu'elle affichait au moment où elle a été embauchée, l'employeur doit s'abstenir de lui nuire dans ses démarches d'affirmation d'elle-même. Surtout, il ne doit pas mettre inopinément fin à son emploi en usant de prétextes ou, comme on le voit encore trop souvent, en «montant un dossier » contre elle.

Quatrièmement, afin de respecter les histoires de vie des personnes trans*, les organisations doivent aussitôt que possible ajuster leurs documents administratifs pour que s'y reflètent les noms, prénoms, genres et photos associées désirés par la personne trans*.

Cinquièmement, les employeurs ne doivent en aucun cas nuire à la confidentialité des informations personnelles de leurs employés trans*. Ils ne doivent pas, par exemple, révéler à qui que ce soit (autres employés, autres employeurs, sous-traitants, etc.) le sexe biologique assigné à la naissance ou encore les noms et prénoms attribués à la naissance, sauf si cela est permis par la loi et avec la permission explicite de la personne concernée par cette information.

Sixièmement, lorsqu'une personne trans* décide de vivre sa transition en cours d'emploi, les employeurs doivent tout faire pour l'accommoder (en lui offrant, par exemple, un congé pour raisons personnelles afin de subir les interventions requises et en veillant à ce que les programmes d'assurance à l'intention du personnel couvrent bien le paiement des hormones, les chirurgies de réassignation sexuelle et tous les autres traitements requis [chirurgie dite esthétique, etc.]).

Septièmement, à son retour au travail, les employeurs doivent, avec l'accord de la personne trans* concernée, faciliter sa réinsertion en emploi en prenant soin de sensibiliser leurs employés (incluant les gestionnaires) à la réalité de la personne trans* et en demandant leur collaboration pleine et entière. Cette démarche de sensibilisation doit aussi rejoindre toutes les personnes potentiellement en lien avec la personne trans* concernée (clients, fournisseurs, sous-traitants, etc.).

Huitièmement, dans certains cas il pourra être indiqué d'aller plus loin que simplement la diffusion d'information. On pourra, par exemple, mettre en place une campagne de sensibilisation à la diversité en milieu organisationnel où l'on démystifiera, entre autres choses, la question de l'identité de genre et de l'expression de genre.

Neuvièmement, dans un contexte de dotation du personnel, nous sommes d'avis que les employeurs doivent persister dans leur volonté d'évaluer la candidature de toutes les personnes intéressées à travailler pour eux sur la base de leurs compétences réelles en lien avec le poste à combler. Dit autrement, est-ce que le candidat « $\mathrm{X}$ » possède réellement les savoirs, les savoir-faire et les savoir-être nécessaires pour répondre aux exigences du poste? En d'autres mots, ils ne devraient JAMAIS chercher à s'enquérir de l'identité de genre ou de l'expression de genre des candidats à un emploi ni même considérer cet élément parmi les critères de sélection du personnel en demandant, par exemple, aux candidats de fournir une photo d'eux-mêmes.

Enfin, dixièmement, il est tout aussi important que les employeurs s'engagent à refuser de considérer 
l'identité de genre et l'expression de genre au moment de déterminer la rémunération de leurs employés, de fixer leurs règles de promotion et, notamment, de rétrogradation.

\subsection{Responsabilités des syndicats ${ }^{39}$}

Comme pour l'État et les employeurs, les syndicats ont aussi un rôle à jouer pour défendre et faire la promotion des droits des personnes trans* sur le marché du travail.

Les syndicats sont traditionnellement reconnus pour deux choses : leur capacité à améliorer les conditions de travail de leurs membres et contribuer au progrès social dans les communautés où ils sont implantés. En ce sens, ils occupent eux aussi une place privilégiée dans la société et ce sont souvent eux, de concert avec les employeurs, qui sont aux premières loges pour témoigner et éventuellement contrer les divers phénomènes de violence dont les personnes trans* sont l'objet. Ils doivent donc, à notre avis, faire diverses choses pour aider les personnes trans* à vivre normalement.

Premièrement, il nous semble fondamental que tous les syndicats adoptent un code de conduite à l'intention de tous leurs membres (incluant leurs gestionnaires et leurs employés) qui stipule que l'organisation ne démontrera aucune tolérance pour tout acte de violence (microagressions et autres) commis à l'égard d'une personne trans* (que cette dernière soit une membre ou une employée de l'organisation, une fournisseuse de services, une sous-traitante ou autre). Comme les collectifs de travail sont parfois le siège de nombreuses microviolences à l'égard des personnes trans*, les syndicats ont la responsabilité de s'assurer que de tels comportements soient clairement reconnus comme étant inacceptables et passibles de sanctions disciplinaires.

Deuxièmement, en relation avec leurs membres (actuels et éventuels), les syndicats ont aussi une certaine responsabilité. Sans pouvoir « contrôler » directement les comportements de leurs membres, ils n'en ont pas moins la possibilité de les influencer de manière positive. À titre d'exemple, si un membre menace le syndicat de déchirer sa carte de membership parce qu'il prend position en faveur des personnes trans*, le syndicat peut lui expliquer à quel point il est important de respecter tous les gens, peu importe leur identité et leur expression de genre. À la fin, si le membre décide de cesser ses relations avec le syndicat, il ne pourra bien entendu rien y faire. Pour contrer tout de même une telle éventualité, l'organisation syndicale pourra prendre les devants et annoncer son intention de développer sa capacité à valoriser toutes les diversités de la main-d'œuvre et, ainsi, préparer psychologiquement ses membres actuels et éventuels à côtoyer des personnes trans*. Cela ne pourra, à notre avis, qu'aider l'organisation syndicale à développer son membership en attirant de nouveaux membres eux-mêmes ouverts à cette diversité. Dans un contexte de batailles syndicales pour préserver leur membership, cela nous semble être une piste très intéressante à explorer.

Troisièmement, lorsque les syndicats comptent dans leurs rangs une personne trans* qui leur annonce son intention de faire sa transition d'un genre à l'autre (ou de se vêtir de temps à autre dans un genre qui n'est pas celui dans lequel elle s'exprimait précédemment), on doit s'abstenir de lui nuire dans ses démarches. Surtout, on ne doit pas mettre inopinément fin à son emploi ou à son statut de membre en usant des prétextes ou en «montant un dossier » contre elle.

Quatrièmement, afin de respecter les histoires de vie des personnes trans*, les organisations syndicales doivent aussitôt que possible ajuster leurs documents administratifs pour que s'y reflètent les noms, prénoms, genres et photos associées désirés par la personne trans*.

Cinquièmement, les syndicats ne doivent en aucun cas nuire à la confidentialité des informations personnelles de leurs employés et membres trans*. Ils ne doivent pas, par exemple, révéler à qui que ce soit (autres employés ou membres, autres employeurs ou syndicats, sous-traitants, etc.) le sexe biologique assigné à la naissance ou encore les noms et prénoms attribués à la naissance, sauf si cela est permis par la loi et avec la permission explicite de la personne concernée par cette information.

Sixièmement, lorsqu'une personne trans* décide de vivre sa transition en cours d'emploi, les syndicats doivent tout faire pour l'accommoder (en lui offrant, par exemple, un congé pour raisons personnelles afin de subir les interventions requises et en veillant à ce que les programmes d'assurance à l'intention du personnel couvrent bien le paiement des hormones, les chirurgies de réassignation sexuelle et tous les autres traitements requis par les personnes trans [chirurgie dite esthétique, etc.]). 
Septièmement, à son retour au travail les syndicats doivent, avec l'accord de la personne trans* concernée, faciliter sa réinsertion en emploi en prenant soin de sensibiliser leurs employés et membres actuels à la réalité de la personne trans* et en demandant leur collaboration pleine et entière. Cette démarche de sensibilisation doit aussi rejoindre toutes les personnes potentiellement en lien avec la personne trans* concernée (clients, fournisseurs, sous-traitants, etc.).

Huitièmement, dans certains cas il pourra être indiqué d'aller plus loin que simplement la diffusion d'information. On pourra, par exemple, mettre en place une campagne de sensibilisation à la diversité en milieu organisationnel où l'on démystifiera, entre autres choses, la question de l'identité de genre et de l'expression de genre.

Neuvièmement, dans un contexte de dotation du personnel, nous sommes d'avis que les syndicats doivent persister dans leur volonté d'encadrer la façon dont le recrutement et la sélection du personnel sont faits par les employeurs et s'assurer que la candidature de toutes les personnes intéressées à travailler pour eux et l'employeur soit considérée sur la base de leurs compétences réelles en lien avec le poste à combler. Dit autrement, est-ce que le candidat « $\mathrm{X}$ » possède réellement les savoirs, les savoir-faire et les savoir-être nécessaires pour répondre aux exigences du poste? En d'autres mots, on ne devrait JAMAIS chercher à s'enquérir de l'identité de genre ou de l'expression de genre des candidats à un emploi ni même considérer cet élément parmi les critères de sélection du personnel en demandant, par exemple, aux candidats de fournir une photo d'eux-mêmes.

Dixièmement, il faut se rappeler que les organisations syndicales sont des institutions démocratiques où le vote de la majorité occupe une place fondamentale quand vient le temps de déterminer les agendas de négociation des conventions collectives. Comme la proportion de gens trans* dans la population générale n'est pas très élevée, on ne peut donc pas attendre qu'une vaste quantité de membres trans* de l'organisation syndicale se regroupent avant de mettre de l'avant des revendications liées au respect de leurs droits.

Pour aider tous les membres de l'organisation syndicale à bien comprendre la réalité des personnes trans*, nous sommes d'avis que l'une des premières choses à faire est de mettre en place un comité pour la reconnaissance de la diversité de la main-d'œuvre (diversité qui pourra inclure, bien évidemment, des gens concernés par les personnes trans*, mais aussi tous les gens aux autres orientations sexuelles, les minorités visibles, les personnes handicapées, etc.). Les recherches empiriques ont démontré que les problèmes dont font face de nos jours les personnes trans* sont les mêmes qu'ont dû affronter, dans le passé, les femmes, les personnes des minorités visibles, les personnes handicapées, etc. Chargées de réfléchir à diverses revendications qu'elles souhaiteraient voir inclure dans les conventions collectives, ces personnes pourraient contribuer à faire en sorte, entre autres choses, que le recrutement et la sélection de la main-d'œuvre se fassent sur des critères beaucoup plus objectifs, comme nous le disions précédemment.

De nos jours, la plupart des conventions collectives sont muettes sur le processus détaillé et les outils de recrutement et de sélection du personnel que peuvent utiliser les employeurs. Cela est, selon nous, une formidable porte d'entrée afin de contribuer à faire en sorte que les droits des personnes faisant partie des minorités soient pleinement reconnus et qu'on cesse enfin, à leur égard, toutes les formes de discrimination dont elles sont encore victimes.

\section{Conclusion}

La question qui consiste à savoir ce qu'est une personne trans* est éminemment complexe. Les personnes trans* ne sont pas des pécheurs qu'il faut punir, des malades mentaux qu'il faut soigner pour leur redonner la raison ou, encore, des semblants de femme produits par une société patriarcale elle-même malade. Les personnes trans* sont des êtres humains parfaitement sains de corps et d'esprit et, de surcroit, elles sont nées comme cela et elles ont toujours existé, partout ${ }^{40}$. On ne choisit pas plus d'être «noir», «femme» ou «vieux» qu'on choisit d'être trans*. Nous sommes ce que nous sommes... c'est tout. Malheureusement, les personnes trans* sont généralement mal comprises et elles sont, en conséquence, victimes de beaucoup de violence et d'énormément de discrimination sur le marché du travail. Nombreuses sont les personnes trans* qui doivent ainsi vivre cachées pour ne pas subir l'opprobre de certains de leurs concitoyens (sic). Pour cette raison, notamment, de nombreuses formations leur sont aujourd'hui offertes pour qu'elles apprennent à développer leurs capacités de résilience. Cela étant dit, si les personnes trans* ont l'occasion d'évoluer dans des milieux sociaux qui les acceptent et les soutiennent 
dans leur cheminement, les recherches démontrent qu'elles sont tout à fait capables d'avoir une vie normale et d'être aussi heureuses que n'importe qui d'autre ${ }^{41}$. Heureusement, les sociétés évoluant grâce à la hausse du niveau d'éducation et la diminution du poids de certaines autorités, leurs droits ont commencé à s'accroître, particulièrement au Québec. Toutefois, nous sommes encore loin de la coupe aux lèvres. Si plusieurs pistes de solution sont envisageables, force est de reconnaitre que très peu semblent actuellement implantées. Pour que ces dernières s'enracinent, nous sommes d'avis que les principaux acteurs du marché du travail doivent prendre leur propre part de responsabilité et qu'ils coopèrent pour non seulement défendre et promouvoir les droits des personnes trans*... mais aussi s'assurer, enfin, qu'elles puissent contribuer au marché du travail à la pleine hauteur de leurs compétences. Tant que cela ne sera pas une réalité, nous ne pourrons affirmer que nous avons su aller au-delà des peurs et de la binarité inégalitaire.

\section{NOTES}

1 Cet article s'ancre dans la réalité du Québec, en ce début de XXIe siècle. Sa visée est essentiellement pragmatique. En d'autres mots, elle ne s'intéresse pas du tout, par exemple, à la question de savoir si la popularisation croissante du phénomène est, ou non, l'un des traits caractéristiques du postmodernisme. Le lecteur intéressé à explorer cette dernière possibilité est invité à lire Felski (2006).

2 Nous sommes conscients que la définition que nous proposons rencontrera vraisemblablement beaucoup de résistance au sein de la société civile. À l'été 2017, publiant dans le journal Le Devoir un article visant à célébrer le premier anniversaire de l'adoption de la modification à l'article 10 de la Charte des droits et libertés de la personne pour y inclure comme motif illicite de discrimination l'identité de genre et l'expression de genre, certains lecteurs ont jugé pertinent de remettre en question nos compétences de chercheurs, car nous écrivions des choses allant vraisemblablement à l'encontre de leurs propres convictions. Le lecteur intéressé à constater ce phénomène peut consulter par lui-même les commentaires émis par certains membres du public pour qui les chercheurs ne devraient pas prendre position dans les débats sociaux en cliquant sur le lien suivant:

https://www.ledevoir.com/opinion/idees/503130/marche-du-travail-la-discrimination-insidieuse-des-trans (page consultée le 14 août 2018). Pour un point de vue opposé, voulant qu'il faille justement que les intellectuels trouvent le courage de prendre position face aux injustices sociales dont sont victimes les personnes trans*, le lecteur pourra lire l'ouvrage de Taylor et HaiderMarkel (2017).

3 Nous entendons par cette expression les pays faisant partie du G7 : Allemagne, Canada, États-Unis, France, Italie, Japon, Royaume-Uni. La Russie, alors qu'elle faisait partie jusqu'en 2014 du G8, faisait déjà bande à part sur la question des droits des personnes issues de la diversité sexuelle et de genre.

4 Certains auteurs préoccupés par ces liens préféreront parler « de cerveau (pour signifier les différences psychologiques entre les sexes), hormones et sexe » (Cossette, 2012).

5 Ces opérations chirurgicales, parfois appelées « opérations de réassignation sexuelle » ou « chirurgies de confirmation de genre » sont encore de nos jours l'objet de nombreuses interrogations et préjugés (Bauquis, Decrouy et Guerid, 2014).

6 Lamoureux (1998) nous explique que les identités peuvent aussi être comprises d'un point de vue social. Dans ce cas de figure, c'est alors la politisation de cette identité qui donnera naissance à divers mouvements sociaux (comme celui des femmes ou des personnes lesbiennes, gaies, bisexuelles, trans et queer). Pour comprendre l'histoire du mouvement trans*, le lecteur est invité à lire Califia (2003) et Valentine (2007).

7 Les femmes trans* (que l'on dit être des femmes nées dans un corps d'homme - Male to Female [ou MTF] selon l'expression américaine consacrée) prendront alors des suppresseurs d'hormones masculines associés à des doses élevées d'hormones féminines qui les aideront à développer leurs seins, répartir leurs graisses et réduire leur pilosité. Les hommes trans* (que l'on dit être des hommes nés dans un corps de femme - Female to Male [ou FTM] selon l'expression américaine consacrée) prendront quant à eux des doses élevées d'hormones masculines qui les aideront à développer leur pilosité et leur masse musculaire tout en aidant leur voix à muer. La plupart des recherches et des récits de vie sur l'histoire individuelle des personnes trans* nous apprennent qu'en général cette identification à l'autre genre se fait très tôt dans la vie... généralement dans la petite enfance ou, au plus tard, au tout début de l'adolescence (Löwy, 2016). Sans savoir pourquoi, les personnes trans* ont souvent eu la conviction d'être nées «dans le mauvais corps » (c'est-à-dire d'être un garçon né dans un corps de fille ou d'être une fille née dans un corps de garçon) (Ames, 2005; Costa et Matzner, 2007).

8 Des quatre catégories que nous utilisons pour définir ce que sont les personnes trans*, l'expression de genre est la seule à l'égard de laquelle la personne a un « certain contrôle naturel». Bien que certaines personnes s'expriment « naturellement» dans un genre qui n’est pas conforme à celui qui leur a été assigné à la naissance, beaucoup de personnes trans* passeront des années à «surjouer » le genre qui leur a été assigné à la naissance pour mieux répondre aux attentes de la société à leur égard. Dans certains cas, les recherches démontrent que c'est plutôt la peur du regard des autres et parfois aussi la honte d'être différent. Cette honte, d'ailleurs, aboutit parfois à la haine de soi-même comme nous le verrons plus tard. Pour comprendre ce phénomène de l'intérieur, le lecteur intéressé pourra consulter les références suivantes : Beck et Speckhard (2013); Bornstein (2012); Jenner (2017); Serano (2007). 
9 Mis à part le cas particulier de certains acteurs comiques comme Robin Williams dans le film Mrs Doubtfire qui portent exceptionnellement des vêtements d'un autre genre l'espace d'une performance artistique, nous ferons généralement référence, ici, à ce que l'on appelle les drag queens et les drag kings. Le lecteur intéressé à en savoir davantage sur ce dernier phénomène pourra lire avec intérêt l'ouvrage de Hastings (2016).

10 Comme nous l'écrivions précédemment, il n’y a aucune relation théorique ni pratique entre le sexe biologique, l’identité de genre, l'expression de genre et l'orientation sexuelle. Si nous avons tenu à présenter ces quatre catégories, ce n'est surtout pas pour insinuer qu'elles permettent d'affirmer que toute personne trans* est, par exemple, homosexuelle. C'est plutôt pour nous aider à comprendre la grande complexité de la question et, surtout, mettre en évidence que dans la pratique les choses sont beaucoup plus complexes que ce qu'elles peuvent sembler au premier abord. En effet, un individu donné peut très bien se trouver à une extrémité d'un continuum catégoriel puis se trouver au milieu des trois autres alors qu'un autre individu peut très bien être au milieu de tous ces continuums catégoriels. Cette dernière remarque nous incite à en faire une autre. Il nous semble prudent de ne pas aborder ces éléments catégoriels comme étant uniquement des « faits déterminés à l'avance » et se trouvant totalement hors du contrôle de la personne humaine. Si cette dernière est probablement « en bonne partie » née comme elle l'est, divers éléments de son environnement et de son propre cheminement sont susceptibles de l'influencer, du moins en partie. Pour être plus clair, bien que cela ne soit qu'une hypothèse qu'il ne nous sera peut-être jamais permis de confirmer, nous croyons que le sexe biologique, l'identité de genre, l'expression de genre et l'orientation sexuelle sont (en partie du moins) sans cesse « construits » et « reconstruits ", aussi bien biologiquement (notamment par les hormones) que socialement (notamment par l'éducation et les diverses pressions sociales), culturellement et politiquement (notamment par des lois et règlements formels et informels régissant les comportements des individus dans un lieu et une époque donnée).

11 Le lecteur intéressé à comprendre comment cette binarité inégalitaire n'est pas, contrairement à ce que l'on propage généralement, le reflet d'une histoire que l'on voudrait naturelle et universelle, lira avec intérêt Espineira, Thomas et Alessandrin (2013); Leroy (2018); Mayor (2014); Weismantel (2013).

12 Le lecteur intéressé à comprendre l'étendue du mouvement réactionnaire s'opposant vertement à la théorie queer pourra lire Perreau (2018).

13 Quelques-uns des ouvrages les plus connus ayant donné une impulsion importante au mouvement queer sont ceux de Michel Foucault (1976; 1984a; 1984b) (sur l'histoire de la sexualité et la société disciplinaire) et de Judith Butler (1990) (sur le trouble dans le genre). Le lecteur intéressé à saisir l'importance de l'œuvre de Butler aura intérêt à lire Baril (2007). Pour une critique de l'œuvre de Butler, le lecteur aura intérêt à examiner les contributions de Namaste (2000 et 2011).

14 Sous l'impulsion du mouvement féministe, les choses ont commencé à changer un peu au cours du XXe siècle, notamment en Occident, mais force est de reconnaitre que les femmes ne sont toujours pas reconnues au même niveau que les hommes. Les études menées à l'échelle internationale le répètent, elles aussi, inlassablement (Banque mondiale, 2003). Au sujet des droits des personnes LGBTQ ailleurs dans le monde, Dorais (2014, p. 169) nous explique que « [...] la montée des fondamentalismes et des intégrismes religieux et de l'extrême droite fait reculer en beaucoup d'endroits les droits des personnes LGBTQ [...] (et que) [s]ur la moitié de la planète, soit environ 90 pays, l'homosexualité est encore un crime passible de sanctions de prison [...] ». Si, au Québec, l'homosexualité n'est plus un crime, il y persiste encore beaucoup d'homophobie (Pellan, 2005).

15 Cette hypothèse, à notre connaissance, n’a encore jamais été validée, du moins au Québec.

16 Les sociologues, soucieux de comprendre ces multiples discriminations qui s'additionnent, parlent alors d'intersectionnalité (Amadieu, 2016). Pour en savoir plus sur cette question, le lecteur est invité à lire Rennes (2016).

17 Pour s'en convaincre, le lecteur pourra lire avec intérêt la contribution de Snorton et Haritaworn (2013) et consulter la page Web suivante consacrée aux personnes trans* assassinées chaque année dans le monde : https://transrespect.org/en/research/ trans-murder-monitoring/.

18 L'histoire de Jeanne d'Arc, condamnée à mourir sur le bûcher, n'en est qu'un exemple parmi d'autres. Parmi les chefs d'accusation portés contre elle, on se rappellera le reproche d'avoir porté, sans nécessité, des vêtements d'homme. En 1580, Montaigne raconte, dans Voyage en Italie, qu'une femme y fut pendue pour s'être habillée en homme. Au sujet de la Seconde Guerre mondiale, on se souviendra que les nazis ont déporté vers les camps de la mort des milliers d'individus suspectés d'être homosexuels. Enfin, à une époque encore plus récente, on se rappellera les actes d'une barbarie innommable commis au milieu des années 2010 par des membres de Daech qui n’hésitaient pas, pendant leur occupation de la Syrie, à attacher à des chaises des personnes prétendument homosexuelles avant de les précipiter en bas des toits d'édifices à étages (repéré à https://www.parismatch.com/Actu/International/Des-enfants-vendus-crucifies-enterres-vivants-703726).

19 Diagnostic and Statistical Manual - Fifth Edition (DSM-V) - Manuel diagnostic des troubles mentaux (5e éd.). Dans un certain nombre de pays, on utilise, en lieu et place de cet ouvrage, la Classification internationale des maladies (CIM). Par souci de concision, toutefois, nous avons choisi de ne pas en traiter. Il est intéressant de remarquer que jusqu'en 1973 l'homosexualité y était encore considérée comme un trouble mental (Dorais, 2014).

20 Il faut savoir que l'étiologie de ce trouble est à ce jour inconnue et qu'aucune théorie satisfaisante ne semble pouvoir l'expliquer (Bourgeois, 2011; WPATH, 2013). Devant cette incapacité de la science à expliquer l'origine de ce phénomène, certains ont donc émis l'hypothèse que ce ne serait donc pas un trouble mental, mais plutôt l'une des manifestations naturelles de la diversité humaine. 
21 L'ouvrage de Karasic et Drescher (2005) est d'ailleurs éloquent à ce sujet. Pour certaines critiques qui se sont penchées sur l'ouvrage de ces auteurs, il semblerait que ce dernier suggère que le DSM est beaucoup plus préoccupé par la préservation et la promotion d'une conception conservatrice des notions de sexe et de genre que par la rigueur scientifique.

22 Dans la précédente version de cet ouvrage (le DSM IV), la question était plutôt abordée sous l'angle du trouble de l'identité de genre. De l'avis de plusieurs auteurs, c'est notamment par souci de « rendre moins pathologique » cette question que les termes utilisés ont été modifiés. Cherchant à « dépathologiser » les personnes trans*, un comité de travail de la World Professional Association for Transgender Health (WPATH) a publié en 2010 un rapport expliquant que le fait d'être une personne trans* n'était pas une maladie en soi. En fait, ce n'est pas le fait d'être trans* qui entraîne des problèmes de santé mentale comme l'anxiété et la dépression. C'est plutôt le fait de vivre dans une société aux genres normés qui stigmatise en toute impunité les personnes dont l'identité de genre et l'expression de genre diffèrent des attentes sociales (repéré à https://tgeu.org/ issues/health_and_depathologisation/depathologisation-health_and_depathologisation/). Nous reviendrons sur ces éléments un peu plus loin dans notre article.

23 Voir, à ce sujet, le lien suivant : http://www.zinzinzine.net/militant-es-trans-ne-jetez-pas-les-personnes-folles-sous-le-bus.html.

24 Par souci de concision, nous n'entrerons pas dans le détail des plans de traitement excessivement complexes et personnalisés en fonction de chacune des personnes trans* concernées, car de nombreuses variables (notamment le lieu et l'époque où le diagnostic est posé; les ressources législatives, médicales et financières à la disposition des personnes trans* concernées; leurs propres caractéristiques physiques et sociales; etc.) doivent alors être prises en compte. Le lecteur intéressé à en savoir davantage sur le sujet aura intérêt à lire le document intitulé Standards de soin pour la santé des personnes transsexuelles, transgenres et de genre non conforme disponible gratuitement à l'adresse suivante : https://www.wpath.org/publications/soc. Il pourra également consulter le site Internet suivant qui constitue un exemple parmi d'autres de cliniques médicales privées dédiées aux soins des personnes trans* $:$ http://www.drchristinemcginn.com/.

25 Pour plus de détails sur l'œuvre controversée de Raymond, nous référons le lecteur à son propre site Internet où elle a senti le besoin de clarifier sa pensée et répondre à ses nombreux détracteurs : http://janiceraymond.com/fictions-and-facts-about-thetranssexual-empire/. Pour une vision féministe radicale et les solutions mises de l'avant pour adresser la question du genre, nous invitions le lecteur à examiner le lien suivant : https://gendertrender.wordpress.com/2013/08/21/authenticity-of-the-forbiddendiscourse-the-silencing-of-feminist-critique-of-gender-statement-has-been-confirmed/. Enfin, pour constater à quel point, encore de nos jours, les femmes trans* ne sont toujours pas bien comprises, acceptées et considérées comme des citoyennes à part entière, même au Québec et par certaines personnes très éduquées, le lecteur est invité à lire le document suivant écrit en décembre 2017 par Denise Bombardier alors qu'elle venait d'apprendre qu'une femme trans* allait présider les destinées de la Fédération des femmes du Québec : https://www.journaldemontreal.com/2017/12/01/une-transgenre-preside-la-ffq.

26 Pour consulter une critique célèbre de l'ouvrage de l'ouvrage de Raymond, nous invitons le lecteur à examiner le lien suivant : https://sandystone.com/empire-strikes-back.html.

27 Cela ne veut pas dire, bien entendu, que les autorités politiques de certains pays, du moins à certaines époques, n'ont pas essayé de nier ce fait. Souvenons-nous, par exemple, du président Ahmadinejad qui affirmait, en 2007, qu'il n'y avait aucun homosexuel en Iran (repéré à http:/ /www.liberation.fr/planete/2007/09/25/ahmadinejad-il-n-y-a-pas-d-homosexuels-en-iran_7821). Pour approfondir la question des droits des personnes LGBTQ dans les pays musulmans, le lecteur lira avec intérêt Jama (2010).

28 Cette variation «naturelle » vient donc s'ajouter à la variation de la couleur de la peau, des cheveux, des yeux, de la morphologie et des traits psychologiques (pour ne parler que de ces éléments). De surcroit, il est important de réaliser que les gens ne choisissent pas la plupart de leurs caractéristiques. Ils naissent ainsi. Ce sont plutôt les membres de la société dans laquelle les personnes trans* évoluent qui leur font croire, à certaines époques, que certaines de leurs caractéristiques sont plus ou moins désirables. À notre avis, c'est lorsque des traitements défavorables sont encouragés sur la base de ces caractéristiques que l'on voit apparaittre, notamment, des plaies comme le racisme, le sexisme, l'âgisme, l'homophobie et la transphobie.

29 Ces différentes formes de violence sont parfois regroupées sous l'appellation «stress de minorité ». Pour visionner des témoignages poignants de ces divers types de violence dont sont victimes les personnes trans*, autant sur le marché du travail que dans leur vie personnelle, le lecteur est invité à consulter Nangeroni et MacKenzie (2009) et Arnold et Schoen (2012).

*30 Nous avons choisi d'inclure dans ce tableau l'automutilation et le suicide, car de nombreuses recherches portant sur les personnes trans* suggèrent que ce sont des violences autoadministrées résultant souvent de multiples pressions sociales qui ont pour effet de terroriser les personnes trans* et de réduire leur espérance de vie (Spade, 2011).

31 Si ces initiatives sont louables à court terme, nous avons la conviction que plusieurs choses plus substantielles doivent impérativement être faites pour ne pas avoir à se rabattre sur des accompagnements aussi personnalisés qui pourraient laisser croire aux personnes trans* que c'est «normal » de devoir faire face à autant de violences et que les seules pistes de solution envisageables sont personnelles.

32 Les variations dans les pourcentages traduisent la région du Québec d’où proviennent les personnes trans*.

33 Comme nous le disions précédemment, au Québec cela n'est interdit que depuis le mois de juin 2016. Au sujet des apparences, toutefois, la loi est encore muette. Ce dernier élément qu'il faudrait ajouter à la Charte des droits et libertés de la personne comprend, à notre avis, la beauté dite « extérieure » (aussi dite « beauté du corps), mais aussi « intérieure » (aussi dite « beauté de l'âme »). 
34 Au Québec, ce n'est que depuis 2006 qu’un changement complet de prénom est accordé à des personnes trans*.

35 Au Québec, ce n'est que depuis 2015 que les personnes trans* peuvent changer la mention de leur sexe sans pour autant devoir avoir préalablement subi une opération de réassignation sexuelle.

36 Au Québec, ce n'est que depuis 2015 que les personnes trans* peuvent changer la mention de leur sexe sans pour autant devoir avoir préalablement subi une opération de réassignation sexuelle.

37 Au Québec, les opérations de réassignation sexuelles ont déjà été couvertes par la Régie de l'assurance-maladie du Québec (RAMQ) avant de cesser de l'être. Ce n'est que depuis 2009 que la RAMQ a recommencé à assumer ces frais. Toutefois, à l'heure où ce texte est écrit, le suivi en thérapie précédant cette opération n’est toujours pas assuré (tout comme ne le sont pas, non plus, toutes les autres interventions dites « esthétiques »).

38 Ces deux droits sont intimement liés. Alors que l'égalité juridique (ou égalité de droit) concerne notamment les conditions d'accès, l'égalité sociale (ou égalité de fait) concerne plutôt les résultats (Cornet, 2014). Très peu de recherches, à notre connaissance, ont porté jusqu'ici sur l'égalité sociale des personnes trans* sur le marché du travail.

39 À notre connaissance, aucune étude scientifique en contexte québécois n’a encore été menée sur cette question.

40 Pour paraphraser Larivière (2017, p. 16), nous dirons que « [...] vouloir dissimuler que (les personnes trans* ont) toujours existé (ne peut faire autrement qu') encourage ( $\mathrm{r}$ ) (la transphobie). L'historien a le devoir de rappeler cette vérité pour les siècles passés ».

41 Pour s'en convaincre, le lecteur intéressé pourra écouter le documentaire intitulé American Transgender diffusé en 2012 par National Geographic Television. En contexte québécois la télésérie intitulée Je suis trans, diffusée sur la chaine Moi \& Cie, est également très informative.

\section{RÉFÉRENCES}

Ainsworth, C. (2015, 18 février). Sex redefined - The idea of two sexes is simplistic Biologists now think there is a wider spectrum than that. Nature|News feature. Repéré à https://www.nature.com/news/sex-redefined-1.16943

Aide aux trans du Québec (ATQ) (2016). Statistiques sur les personnes trans*. Repéré à http://www.atq1980.org/archives/articles/ statistiques-sur-les-personnes-transsexuelles/

Amadieu, J.-F. (2002). Le poids des apparences - Beauté, amour et gloire. Paris, France : Odile Jacob.

Amadieu, J.-F. (2006). Les clés du destin - École, amour, carrière. Paris, France : Odile Jacob.

Amadieu, J.-F. (2016). La société du paraître - Les beaux, les jeunes... et les autres. Paris, France : Odile Jacob.

Ames, J. (dir.) (2005). Sexual metamorphosis - An anthology of transsexual memoirs. New York, NY: Vintage Books.

Anteby, M. et Anderson, C. (2014). The shifting landscape of LGBT organizational research. Research in Organizational Behavior, 34, 3-25.

Arnold, C. et Dr. Schoen, M. (2012). Trans - It takes a special kind of courage to be who you really are [livre audio].

Banque mondiale (2003). Genre et développement économique - Vers l'égalité des sexes dans les droits, les ressources et la participation. Washington, DC: Banque mondiale.

Barbash, J. (1984). The elements of industrial relations. Madison, WI: University of Wisconsin Press.

Baril, A. (2007). De la construction du genre à la construction du "sexe" : Les thèses féministes postmodernes dans l'œuvre de Judith Butler. Recherches féministes, 20(2), 61-90.

Bauer, G. R., Hammond, R., Travers, R., Kaay, M., Hohenadel, K. M. et Boyce, M. (2009). I don't think this is theoretical; This is our lives: How erasure impacts health care for transgender people. Journal of the Association of Nurses in Aids Care, 25(3), $348-361$.

Bauquis, O., Decrouy, V. et Guerid, S. (2014). La chirurgie de réassignation sexuelle : De l'ignorance au préjugé. Forum Med Suisse, 14(49), 919-923.

Beck, K. et Speckhard, A. (2013). Warrior Princess - A U.S. navy SEAL's journey to cming out transgender. McLean, VA: Advances Press.

Becker, J. V. et Perkins, A. (2014). Gender dysphoria. Dans The American Psychiatric Publishing Textbook of Psycbiatry (6e éd.). 
Bell, M. P., Ozbilgin, M. F., Beauregard, T. A. et Surgevil, O. (2011). Voice, silence, and diversity in 21st century organizations: Strategies for inclusion of gay, lesbian, bisexual and transgender employees. Human Resource Management, 50(1), 131-146.

Bender-Baird, K. (2011). Transgender employment experiences - Gendered perceptions and the law. Albany, NY: SUNY Press.

Bornstein, K. (2012). A queer and pleasant danger - A memoir. Boston, MA: Beacon Press.

Bourcier, M.-H. (2006). Queer zones - Politique des identités sexuelles et des savoirs. Paris, France : Éditions Amsterdam.

Bourgeois, M. (2011). Le spectre des dysphories de genre. Dans A. Allessandrin (dir), La transidentité - Des changements individuels au débat de société. Paris, France : L’Harmattan.

Bourré, J.-S. (2017). TRANSition - Évolution du mouvement trans et de ses revendications. Candiac, Québec : Marcel Broquet Éd.

Butler, J. (1990). Gender trouble: Feminism and the subversion of identity. New York, NY: Routledge.

Califia, P. (2003). Le mouvement transgenre - Changer de sexe. Paris, France : EPEL.

Calvez, V. (2009). Discrimination et gestion de la diversité : Au carrefour des peurs dans l'organisation. Comparaisons internationales. Humanisme \& Entreprise, 295, 1-15.

Cardon, P. (2009). Post-queer : Pour une « approche trans-genre » ou le trans-genre comme catégorie d'analyse. Diogène, 225, 172-188.

Chicha, M.-T. et Saba, T. (dir.) (2010). Diversité en milieu de travail : Défis et pratiques de gestion. Montréal, Québec : HEC Montréal.

Colpron, S. (2016, 6 juin). La loi Olie. La Presse, p. 6.

Conseil québécois LBGT (2017). Politiques transversales : Revendications pour un Québec trans-inclusif. Montréal, Québec : Comité trans du Conseil québécois LGBT.

Conway, L. (2002). How frequently does transsexualism occur? Repéré à http://ai.eecs.umich.edu/people/ conway/TS/TSprevalence.html

Corcos, M. (2015). Qui a peur de la maladie mentale? 10 bonnes raisons de se méfier du DSM 5. Paris, France : Dunod.

Cornet, A. (2014). L’Approche intégrée du genre dans l'élaboration des politiques socio-économiques. Regards croisés sur l'économie, 15, 52-68.

Cossette, L. (dir.) (2012). Cerveau hormones et sexe - Des différences en question. Montréal, Québec : Éditions du remue-ménage.

Costa, L. R. et Matzner, A. (2007). Male bodies women's souls - Personal narratives of Thaïland's transgendered youth. Binghamton, NY: Haworth Press.

Cox, jr., T. (1994). Diversity in organizations - Theory, research and practice. San Francisco, CA: Barrett-Koehler Publishers.

Davis, H. F. (2017). Beyond trans - Does gender matter? New York, NY: New York University Press.

Davy, Z. (2011). Recognizing transsexuals - Personal, political and medicolegal embodiment. Burlington, VT: Ashgate Publishing Company.

Desjardins, P. (2013). Le DSM-5 et l'évaluation des troubles mentaux. Psychologie Québec/Pratiques professionnelles, 30(6), 8-11.

Dorais, M. (2014). De la honte à la fierté - 250 jeunes de la diversité sexuelle se révèlent. Montréal, Québec : VLB Éditeur.

Ekins, R. et King, D. (2006). The transgender phenomenon. Londres, Royaume-Uni, Sage Publications.

Erickson-Schroth, L. et Jacobs, L. A. (2017). You're in the wrong batbroom - And 20 other myths and misconceptions about transgender and gender - Nonconforming people. Boston, MA: Beacon Press.

Espineira, A., Thomas, M.-Y. et Alessandrin, A. (2013). La transidentité en 2011. Dans M.-Y. Thomas, K. Espineira et A. Alessandrin (dir.), Transidentités - Histoire d'une dépathologisation (p. 127-132). Paris, France : L'Harmanttan.

Feinberg, L. (1996). Transgender warriors-making history from Joan of Arc to Dennis Rodman. Boston, MA: Beacon Press. 
Felski, R. (2006). Fin de siècle, fin du sexe - Transsexuality, postmodernism, and the death of history. Dans S. Striker et S. Whittle (dir.), The transgender studies reader (p. 565-573). New York, NY: Routledge.

Foucault, M. (1976). Histoire de la sexualité (Tome I - La volonté de savoir). Paris, France: Gallimard.

Foucault, M. (1984a). Histoire de la sexualité (Tome II - L'usage des plaisirs). Paris, France: Gallimard.

Foucault, M. (1984b). Histoire de la sexualité (Tome III - Le souci de soi). Paris, France: Gallimard.

Gibney, E. (2016). Excluded, intimidated and harassed: LGBT physicists face discrimination - Transgender people are the most affected. Nature|Trend Watch, 22(mars).

Gioia, D. A., Hamilton, A. L. et Patvardhan, S. D. (2014). Image is everything: Reflections on the dominance of image in modern organizational life. Research in Organizational Behavior, 34, 129-154.

Goguel d'Allondans, T. (2017). Ados LBGTI - Les mondes contemporains des jeunes lesbiennes, gays, bisexuel(le)s, transgenres, intersexes. Québec, Québec : Presses de l'Université Laval.

Gouvernement du Québec (2014). Réalités juridiques et sociales des minorités sexuelles dans les principaux pays d'origine des personnes nouvellement arrivées an Québec. Québec, Québec : ministère de l'Immigration, de la Diversité et de l'Inclusion.

Gouvernement du Québec (2013). Les pratiques de recrutement des entreprises au Québec. Québec, Québec : ministère de l'Emploi et de la Solidarité sociale.

Grand dictionnaire terminologique de la langue française (GDTLF) (2016). Repéré à http://gdt.oqlf.gouv.qc.ca / ficheOqlf.aspx?Id Fiche $=8370125$

Grant, J. M., Mottet, L. A., Tanis, J., Harrison, J., Herman, J. L. et Keisling, M. (2011). Injustice at every turn: A report of the national transgender discrimination survey. Executive summary. Washington, DC: National Center for Transgender Equality and National Gay and Lesbian Task Force.

Guénette, A. M. et Le Garrec, S. (dir.) (2016). Les peurs au travail. Toulouse, France: Octares.

Hall, D. E., Jogose, A.-M., Bebell, A. et Potter, S. (dir.) (2013). The Routledge queer studies Reader. New York, NY: Routledge.

Harrington, L. (2016). Traversing gender - Understanding transgender realities. Anchorage, AK: Mystic Productions Press.

Hastings, M. (2016). Why Drag? San Francisco, CA: Chronicle Books.

Heller, M. (2006). More employers broadening nondiscrimination policies to include transgender workers. Workforce Management, 85(12), 62.

Hill, R. J. (2009). Incorporating queers: Blowback, backlash, and other forms of resistance to workplace diversity initiatives that support sexual minorities. Advances in Developing Human Resources, 11(1), 37-38.

Hines, S. et Sanger, T. (dir) (2010). Transgender identities - towards a social analysis of gender diversity. New York, NY: Routledge.

Holck, L., Muhr, S. L. et Villesèche, F. (2016). Identity, diversity and diversity management: On theoretical connections, assumptions and implications for practice. Equality, Diversity and Inclusion: An International Journal, 35(1), $48-64$.

Jama, A. (2010). Citoyens interdits - Les minorités sexuelles dans les pays musulmans. Saint-Martin-de-Londres, France : H \& O.

Jenner, C. (2017). The secrets of my life. New York, NY: Grand Central Publishing.

Jordan-Young, R. M. (2016). Hormones, sexe et cerveau. Paris, France: Belin.

Karasic, D. et Drescher, J. (dir.) (2005). Sexual and gender Diagnoses of the diagnostic and statistical manual (DSM) - A reevaluation. Binghamton, NY: Haworth Press.

Klie, S. (2009). LGBT employees still face barriers. Canadian HR Reporter, (22), 8.

Lambert, J. R. (2015). The impact of gay-friendly recruitment statements and due process employment on a firm's attractiveness as an employer. Equality, Diversity and Inclusion: An International Journal, 34(6), 510-526.

Lamoureux, D. (dir.) (1998). Les limites de lidentité sexuelle. Montréal, Québec : Éditions du Remue-ménage.

Larivière, M. (2017). Dictionnaire historique des homosexuels célèbres. Paris, France : La Musardine. 
Lawrence, A. A. (2014). Chapter 39. Gender dysphoria. Gabbard's Treatments of Psychiatric Disorders (5e éd.).

Ledford, H. (2016, 7 avril). Door-to-door canvassing reduces transphobia. A simple non-judgemental chat can lessen prejudice towards transgender people. Nature $\mid$ News features.

Leroy, A. (2018). Repolitiser le genre. Alternatives Sud, 25(2), 7-26.

Lipovetsky, G. (2017). Plaire et toucher - Essai sur la société de séduction. Paris, France : Gallimard.

Löwy, I. (2016). Quelques remarques historiques sur des corps atypiques et le sexe/genre du cerveau. Dans R. M. JordanYoung, Hormones, sexe et cerveau (p. 525-532). Paris, France : Belin.

Macé, É. (2010). Ce que les normes de genre font aux corps/Ce que les corps trans font aux normes de genre. Sociologie, 4(1), 497-515.

Maillé, C. (2012). Féminismes, genre et sexe au XXIe siècle : nouveaux habits, vieux débat? Dans L. Cossette (dir.), Cervean hormones et sexe - Des différences en question. Montréal, Québec : Éditions du remue-ménage.

Malo, F. B. (2013). Le recrutement. Dans B. Ancelin (dir), Nouveaux métiers RH - Enjeux et compétences clés de demain (p. 43-78). Quercy, France : Focus RH.

Malo, F. B. (2011). Le recrutement, la sélection et l'accueil du personnel - Outils pour développer les compétences du professionnel. Québec, Québec : PUQ.

Malo, F. B. et Bettache, M. (2016, 11 juillet). La discrimination insidieuse envers les trans. Le Devoir. Repéré à https://www.ledevoir.com/opinion/idees/503130/marche-du-travail-la-discrimination-insidieuse-des-trans

Malo, F. B. (2018) (sous presse). La dotation du personnel trans* dans les organisations québécoises. Dans F. B. Malo, J. D. Thwaites et Y. Hallée (dir.), L'Humain plus qu'une ressource au cœur de la gestion. Québec, Québec : PUL.

Mattilda (connue sous le nom de Matt Bernstein Sycamore) (dir.) (2006). Nobody passes - Rejecting the rule of gender and conformity. Berkeley, CA: Seal Press.

Mayor, A. (2014). Les Amazones - Quand les femmes étaient les égales des hommes (VIIIe siècle av. J.-C. - Ier siècle apr. J.-C.). Paris, France: La Découverte.

McFadden, C. (2015). Lesbian, gay, bisexual, and transgender careers and human resource development: A systematic literature review. Human Resource Development Review, 14(2), 125-162.

Meyer, I. H. (2015). Resilience in the study of minority stress and health of sexual and gender minorities. Psychology of Sexual Orientation and Gender Diversity, 2(3), 209-213.

Montargot, N. et Peretti, J.-M. (2014). Regards de responsables sur les notions d'égalité, non-discrimination et diversité. Management \& Avenir, 68, 183-200.

Montreuil, M. A. H. (2015). Embauche, accueil et intégration d'une transgenre au sein d'une organisation - Aspects éthiques et organisationnels. Document préparé pour le cours Créativité et résolution de problèmes (RLT-7028). Université Laval.

Morency, M-A., Simard, J. et Pépin, R. (2006). Le triple statut des professionnels salariés et son impact sur l'encadrement des praticiens réflexifs. Interventions, 125, 194-204.

Nadal, K. L. (2013). That's so gay! Microagressions and the lesbian, gay, bisexual and transgender community. Washington, DC: American Psychological Association.

Namaste, V. (2000). Invisible lives - The erasure of transsexual and transgendered people. Chicago, IL: University of Chicago Press.

Namaste, V. (2011). Sex change, social change - Reflections on identity, institutions, and imperialism (2e éd.). Toronto, Ontario: Canadian Scholar's Press.

Nangeroni, N. et MacKenzie, G. O. (2009). Transgender at work. [Vidéo en ligne] Gender Education \& Media, Beverly. Repéré à https://archive.org/details/GendervisionTransgenderAtWorkPart1

National Geographic Society (2012). American Transgender. [Vidéo en ligne] National Geographic Television. Repéré à http://natgeotv.com/ca/american-transgender 
Navarro Swain, T. (1998). Au-delà du binaire : Les queers et l'éclatement du genre. Dans D. Lamoureux (dir.), Les limites de l'identité sexuelle. Montréal, Québec : Éditions du Remue-ménage.

Ordre des conseillers en ressources humaines et relations industrielles agréés (OCRHA). Code de déontologie. Montréal, Québec : OCRHA.

Ouellette-Vézina, H. (2018, 8 août). Fierté Montréal mise sur la diversité et la parité. Journal Métro. Repéré à http://journal metro.com/actualites/montreal/1713695/la-12e-edition-du-festival-fierte-montreal-est-lancee/

Page, S. E. (2017). The diversity bonus - How great teams pay off in the knowledge economy. Princeton, NJ: Princeton University Press.

Pellan, G. (2005). L’homophobie - Un comportement hétérosexuel contre nature. Montréal, Québec : Éditions Québec Amérique.

Peretti, M.-L. (2013). La transgenralité, une variable dans la construction de l’identité. Le Journal des psychologues, 310, 58-62.

Peretti, J.-M. (2012a). La gestion de la diversité. Dans J. Allouche (dir.), Encyclopédie des ressources humaines - Théories, instruments, méthodes, auteurs (3e éd., p. 394-400). Paris, France : Vuibert.

Peretti, J.-M. (dir.) (2012b). L'Encyclopédie des diversités. Paris, France : Éditions EMS.

Perreau, B. (2018). Qui a peur de la théorie queer? Paris, France : Presses de Sciences Po.

Pfafflin, F. et Junge, A. (1998). Sex reassignment: Thirty years of international follow up studies after sex reassignment surgery. A comprehensive review 1961-1991. International Journal of Transgenderism. Repéré à https://www.researchgate.net/ publication/299412537 Sex Reassignment Thirty Years of International Follow-up_Studies_after_Sex Reassignment Surgery A Comprehensive Review 1961-1991

Phillips, J M. et Gully, S. M. (2009). Assessing external job candidates. Alexandria, VA: Society for Human Resource Management.

Priola, V., Diego, L., De Simone, S. et Serri, F. (2014). The sound of silence. Lesbian, gay, bisexual and transgender discrimination. Dans Inclusive organizations. British Journal of Management, 25(3), 488.

Raymond, J. G. (1979). The transsexual empire - The making of the she-male: A fascinating look at transsexual operations and the 'cures' they promise. Boston, MA: Beacon Press.

Rennes, J. (dir.) (2016). Encyclopédie critique du genre - Corps, sexualité, rapports sociaux. Paris, France : La Découverte.

Rudin, J., Yang, Y., Ruane, S., Ross, L., Farro, A. et Billing, T. (2016). Transforming attitudes about transgender employee rights. Journal of Management Education, 40(1), 30-46.

Salamon, G. (2010). Assuming a body - Transgender and rhetorics of materiality. New York, NY: Columbia University Press.

Sanger, T. (2010). Beyond gender and sexuality binaries in sociological theory - The case for transgender Inclusion. Dans S. Hines et T. Sanger (dir.), Transgender identities - Towards a social analysis of gender diversity (p.259-276). New York, NY: Routledge.

Sauvé, J.-S. (2015). L'interdiction de discriminer les personnes trans* dans la Charte des droits et libertés de la personne : pour son amélioration par l'ajout de l'identité de genre et de l'expression de genre à la liste des motifs de distinction illicites. Revue internationale Enfances Familles Générations, 23, 108-126.

Schmidt, S. W., Githens, R. P., Rocco, T. S. et Kormanik, M. B. (2012). Lesbian, gays, bisexual, and transgenred people and human resource development: An examination of the literature in adult education and human resource development. Human Resource Development Review, 11(3), 326-348.

Serano, J. (2007). Whipping girl - A transsexual woman on sexism and the scapegoating of feminity. Emeryville, CA: Seal Press.

Singh, A. (2018). The queer and transgender resilience workbook - Skills for navigating sexual orientation \& gender expression. Oakland, CA: New Harbinger Publications.

Sironi, F. (2011). Psychologie(s) des transsexuels et des transgenres. Paris, France : Odile Jacob.

Snorton, C. R. et Haritaworn, J. (2013). Trans necropolitics - A transnational reflection on violence, death, and the trans of color afterlife. Dans S. Stryker et A. Z. Aizura (dir.), The transgender studies reader 2 (p. 66-76). New York, NY: Routledge.

Spade, D. (2011). Normal life - Administrative violence, critical trans, and the limits of law. Brooklyn, NY: South End Press. 
Steinberg, S. (2013). L’anthropologue, les médecins et l'expérience transgenre - Questions posées à Laurence Hérault. Clio. Femmes, Genres, Histoire, 37, 162-176.

Stoller, R. J. (1978). Recherches sur l'identité sexuelle. Paris, France : Gallimard.

Stone, S. (1991). The empire strikes back. Repéré à https://sandystone.com/empire-strikes-back.html

Stryker, S. (2008). Transgender history. Berkeley, CA: Seal Studies.

Taubira, C. (2014). Paroles de liberté. Paris, France : Flammarion.

Taylor, J. K. et Haider-Markel, D. P. (2017). Conclusions and future directions in transgender politics and policy. Dans J. K. Taylor, et D. P. Haider-Markel (dir.), Transgender rights and politics - Groups, issue framing \& policy adoption. Ann Harbor, MI: University of Michigan Press.

Taylor, J. K. et Haider-Markel, D. P. (dir) (2014). Transgender Rights and Politics - Groups, issue framing \& policy adoption. Ann Harbor, MI: University of Michigan Press.

Teitel, E. (2015, 27 avril). The risky state of being trans in public. Maclean's.

Theodorakopoulos, N. et Budhwar, P. (2015). Diversity and inclusion in different work settings: Emerging patterns, challenges and research agenda. Human Resource Management, 54(2), 177-197.

Tosh, J. (2016). Psychology and gender dysphoria - Feminist and transgender perspectives. New York, NY: Routledge.

Totman, R. (2003). The third sex - Kathoey - Thailland's ladyboys. Londres, Royaume-Uni: Souvenir Press.

Trans PULSE (2011). Nous avons du pain sur la planche : la discrimination au travail et les défis d'emploi pour les personnes trans en Ontario. Bulletin électronique de Trans PULSE, 2(1), 1-3.

Tuttle, H. (2015). Developing a strategy for transgender employees. Risk Management, 4-7.

Valentine, D. (2007). Imagining transgender - An ethnography of a category. Durham, NC: Duke University Press.

Wang, P. et Schwarz, J. L. (2010). Stock price reactions to LGBT nondiscrimination policies. Human Resource Management, 49(2).

Weismantel, M. (2013). Towards a transgender archeology - A queer rampage through prehistory. Dans S. Stryker et A. Z. Aizura (dir.), The transgender studies reader 2 (p.319-334). New York, NY: Routledge.

Wilchins, R. (2004). Queer theory, gender theory - An instant primer. Bronx, NY: Magnus Books.

World Professional Association for Transgender Health (WPATH) (2013). Standards de soin pour la santé des personnes transsexuelles, transgenres et de genre non conforme. Repéré à https://www.wpath.org/publications/soc

Yarhouse, M. A. (2015). Understanding gender dysphoria - Navigating transgender issues in a changing culture. Downers Grove, IL: InterVarsity Press. 